\title{
Molecullar Cell
}

\section{Mitochondrial Threonyl-tRNA Synthetase TARS2 Is Required for Threonine-Sensitive mTORC1 Activation}

\section{Graphical Abstract}

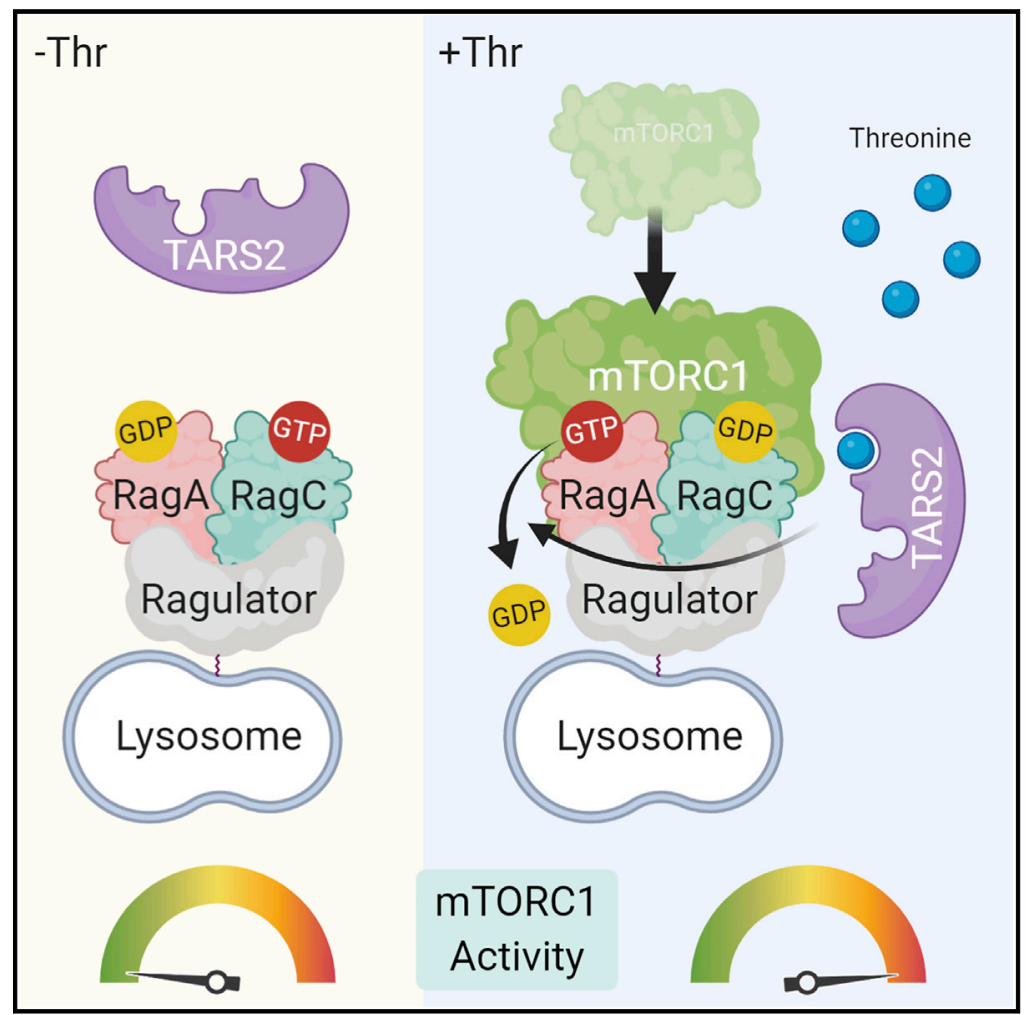

\section{Highlights}

- Mitochondrial protein TARS2 interacts with lysosomal machinery-sensing amino acids,

- Threonine modulates lysosomal localization and activity of mTORC1 through TARS2

- TARS2 binds inactive RagC and elevates GTP loading of RagA in response to threonine

- TARS2 is required for threonine-dependent regulation of the mTORC1 signaling pathway

\section{Authors}

Sung-Hoon Kim, Jung-Hyun Choi, Peng Wang, ..., Anne-Claude Gingras, Seyed Mehdi Jafarnejad, Nahum Sonenberg

\section{Correspondence}

sm.jafarnejad@qub.ac.uk (S.M.J.), nahum.sonenberg@mcgill.ca (N.S.)

\section{In Brief}

Monitoring the abundance of nutrients such as amino acids and regulating the cellular metabolism accordingly are key functions of the mTORC1 signaling pathway. Kim et al. reveal a pathway involving the mitochondrial protein TARS2, which controls mTORC1 activity in response to the changing levels of the amino acid threonine. 


\title{
Short Article
}

\section{Mitochondrial Threonyl-tRNA Synthetase TARS2 Is Required for Threonine-Sensitive mTORC1 Activation}

\author{
Sung-Hoon Kim, ${ }^{1,2}$ Jung-Hyun Choi, ${ }^{1,2}$ Peng Wang, ${ }^{1,2}$ Christopher D. Go, ${ }^{3,4}$ Geoffrey G. Hesketh, ${ }^{3}$ \\ Q1 Anne-Claude Gingras, ${ }^{3,4}$ Seyed Mehdi Jafarnejad, ${ }^{5, *}$ and Nahum Sonenberg $1,2,6,{ }^{*}$ \\ ${ }^{1}$ Goodman Cancer Research Centre, McGill University, Montreal, QC H3A 1A3, Canada \\ 2Department of Biochemistry, McGill University, Montreal, QC H3A 1A3, Canada \\ ${ }^{3}$ Centre for Systems Biology, Lunenfeld-Tanenbaum Research Institute, Sinai Health System, Toronto, ON M5G 1X5, Canada \\ ${ }^{4}$ Department of Molecular Genetics, University of Toronto, Toronto, ON M5S 1A1, Canada \\ ${ }^{5}$ Patrick G. Johnston Centre for Cancer Research, Queen's University Belfast, Belfast BT9 7AE, UK \\ ${ }^{6}$ Lead Contact \\ *Correspondence: sm.jafarnejad@qub.ac.uk (S.M.J.), nahum.sonenberg@mcgill.ca (N.S.) \\ https://doi.org/10.1016/j.molcel.2020.11.036
}

\section{SUMMARY}

Mechanistic target of rapamycin complex 1 (mTORC1) controls cell growth and proliferation by sensing fluctuations in environmental cues such as nutrients, growth factors, and energy levels. The Rag GTPases (Rags) serve as a critical module that signals amino acid (aa) availability to modulate mTORC1 localization and activity. Recent studies have demonstrated how aas regulate mTORC1 activity through Rags. Here, we uncover an unconventional pathway that activates mTORC1 in response to variations in threonine (Thr) levels via mitochondrial threonyl-tRNA synthetase TARS2. TARS2 interacts with inactive Rags, particularly GTPRagC, leading to increased GTP loading of RagA. mTORC1 activity in cells lacking TARS2 is resistant to Thr repletion, showing that TARS2 is necessary for Thr-dependent mTORC1 activation. The requirement of TARS2, but not cytoplasmic threonyl-tRNA synthetase TARS, for this effect demonstrates an additional layer Q2 of complexity in the regulation of mTORC1 activity.

\section{Q3 Q4 Q7 INTRODUCTION}

Mechanistic target of rapamycin complex 1 (mTORC1) is a central regulator of cell growth and proliferation that exquisitely responds to the availability of nutrients, growth factors, and energy levels (Hay and Sonenberg, 2004; Wullschleger et al., 2006; Wolfson and Sabatini, 2017). mTORC1 orchestrates anabolic processes such as protein synthesis (Ben-Sahra and Manning, 2017) and catabolic processes including autophagy (Saxton and Sabatini, 2017), depending on the external stimuli. Dysregulated mTORC1 activity is frequently implicated in diseases such as cancer, diabetes, and neurodegenerative disorders (Efeyan et al., 2012).

Amino acids (aas) regulate mTORC1 activity via the Rag guanosine triphosphatases (Rags) (Hara et al., 1998; Sancak et al., 2010). Rags form a heterodimer of RagA or RagB (RagA/B) and RagC or RagD (RagC/D) (Sekiguchi et al., 2001) and are responsible for aa-dependent mTORC1 activation at the lysosomal surface (Dibble and Cantley, 2015). The aas switch nucleotide loading of Rags via guanine nucleotide exchange factor (GEF) (Bar-Peled et al., 2012; Shen and Sabatini, 2018) or guanosine triphosphatase-activating protein (GAP) (Bar-Peled et al., 2013). The nucleotide state of Rags determines their interaction with Raptor, an exclusive component of mTORC1. Raptor preferentially binds active Rags composed of GTP-RagA/B and
GDP-RagC/D (Sancak et al., 2008). Although some proteins (or protein complexes) have been identified as sensors for a few specific aas-leucine (Leu), arginine, glutamine, and methionine in the mTORC1 pathway (Jewell et al., 2015; Wang et al., 2015; Wolfson et al., 2016; Chantranupong et al., 2016; Gu et al., 2017; Jung et al., 2019)-sensors for other aas remain unknown. Aminoacyl-tRNA synthetases (ARSs) are enzymes that attach aas to their cognate tRNAs. It has been shown that cytoplasmic ARSs function upstream or downstream of mTORC1 (Arif et al., 2017; Luo et al., 2018; Dai et al., 2018). Q8 Importantly, leucyl-tRNA synthetase (LARS) was identified as a Leu sensor in the mTORC1 pathway (Bonfils et al., 2012; Han et al., 2012; He et al., 2018); however, the role of other ARSs in the mTORC1 pathway, particularly as aa sensors, has not been described.

Here, we reveal that mitochondrial (mt) threonyl-tRNA synthetase 2 (TARS2) activates mTORC1 in response to increased threonine (Thr) levels. TARS2 associates with inactive Rags, preferentially with GTP-RagC, and elevates GTP loading of RagA and mTORC1 activity. This activity cannot be achieved by cytoplasmic threonyl-tRNA synthetase (TARS). We further show that TARS2 controls cell proliferation and mRNA translation in a Thr-dependent manner, demonstrating that TARS2 plays a key role in aa signaling and regulation of $\mathrm{mTORC}$ activity. 
A

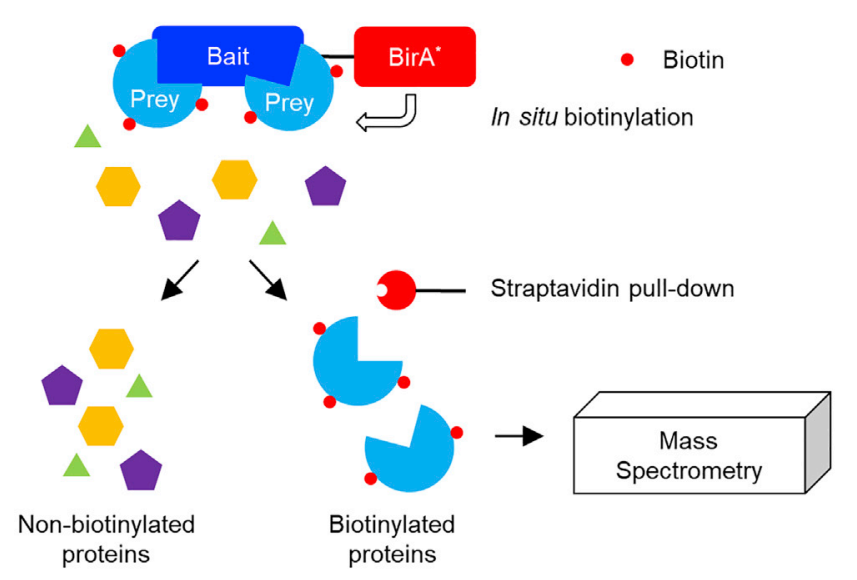

B

HumanCellMap baits

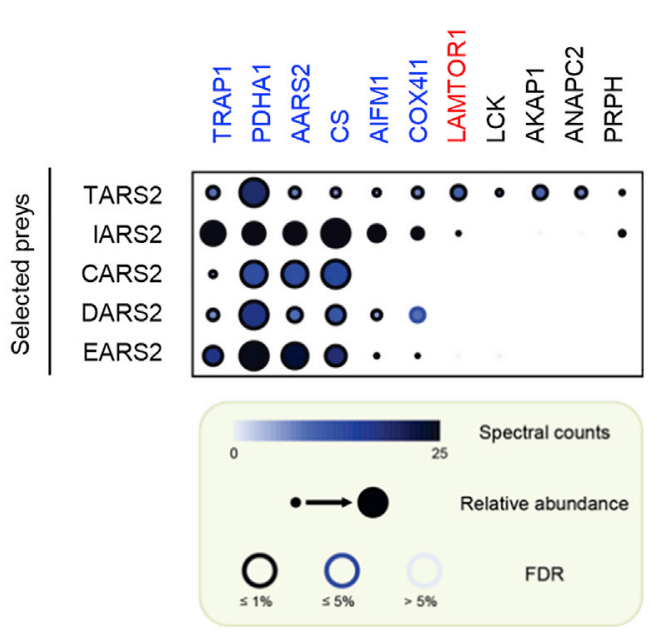

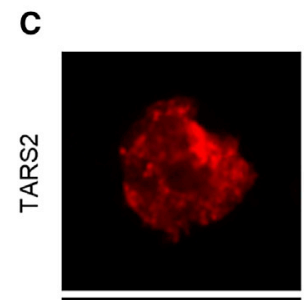
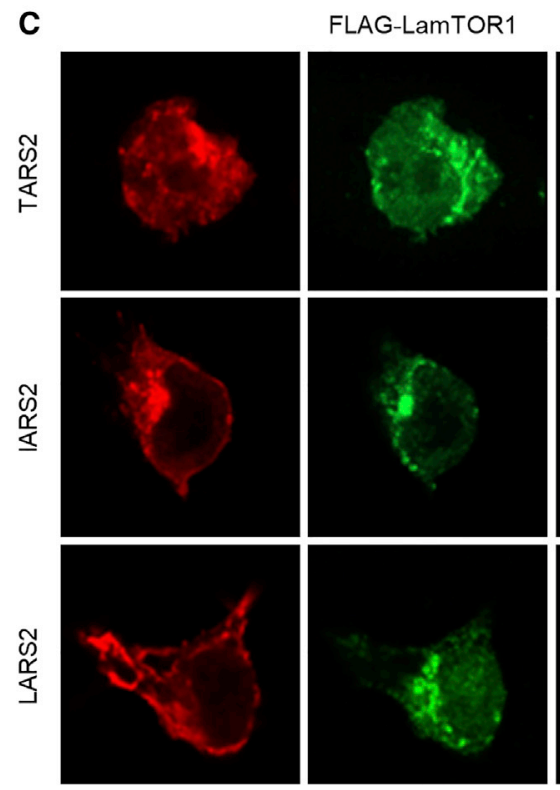
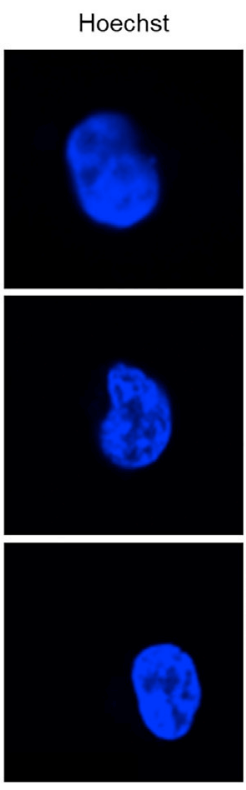
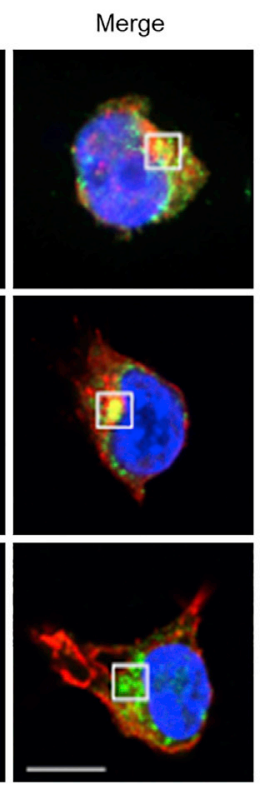

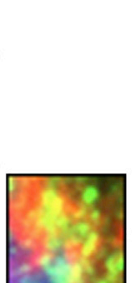

D
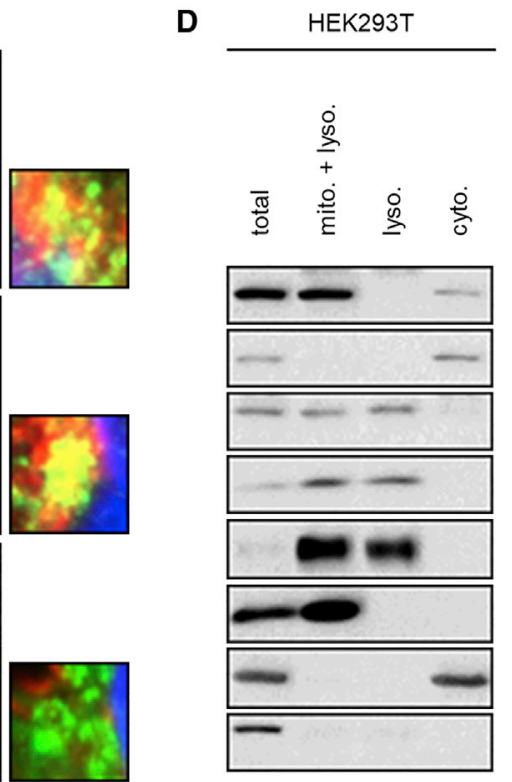

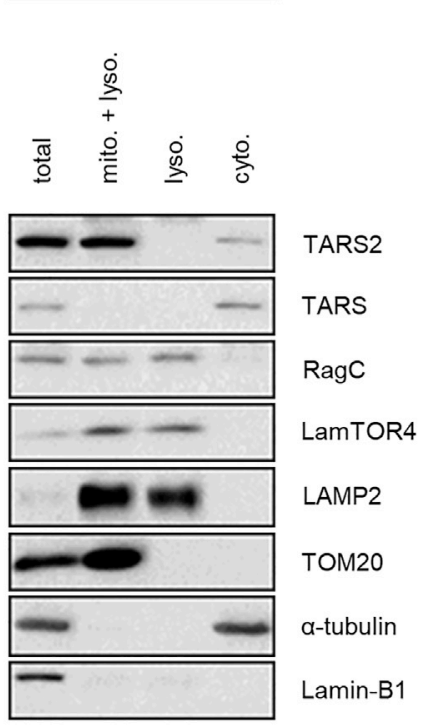

Figure 1. TARS2 Interacts with aa-Sensing Machinery of mTORC1

(A) Schematic workflow of BiolD assay using an abortive biotin ligase BirA R118G (BirA*).

(B) Specific recovery of TARS2 as prey with BirA*-tagged LAMTOR1 as a bait from humancellmap.org. Proximal interaction of the indicated baits with mt-ARSs TARS2, IARS2, CARS2 (cysteinyl-ARS), DARS2 (aspartyl-ARS), and EARS2 (glutamyl-ARS) -is shown. The mt proteins and lysosomal LAMTOR1 are shown in blue and red, respectively.

(C) Subcellular localization of mt-ARSs with LamTOR1 in HEK293T cells. Nuclei were stained by Hoechst (blue). White squares in the merged images are enlarged in the right images. Scale bar $10 \mu \mathrm{m}$.

(D) Subcellular localization of TARS2. The total and mixtures of mitochondria and lysosomes (mito. + lyso.), pure lysosomes (lyso.), and cytoplasmic (cyto.) fractions are shown. LAMP2 (lysosome), TOM20 (mitochondrion), $\alpha$-tubulin (cytoplasm), and Lamin-B1 (nuclei).

See also Figure $\mathrm{S} 1$.

\section{RESULTS AND DISCUSSION}

TARS2 Interacts with aa Sensors in the mTORC1 Pathway Proximity-dependent biotinylation coupled to mass spectrometry (BiolD) is a powerful tool to uncover spatial relationships be- tween proteins (Figure 1A). It enables large-scale projects that involve multiple bait proteins to uncover the organization of individual organelles (Gupta et al., 2015; Youn et al., 2018). We recently documented the protein organization of a human cell through the profiling of 192 subcellular marker proteins (Go 


\section{Molecular Cell}

A

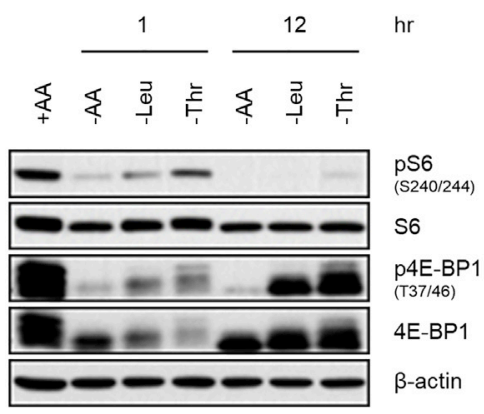

C

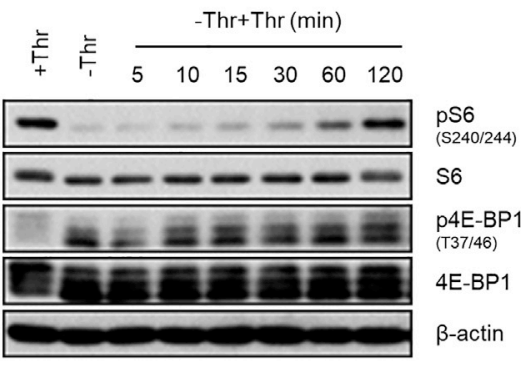

$\mathbf{E}$

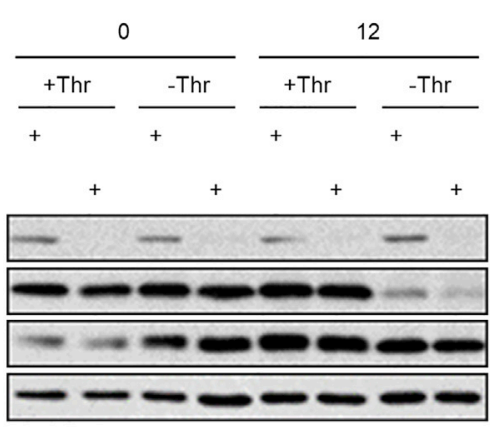

B

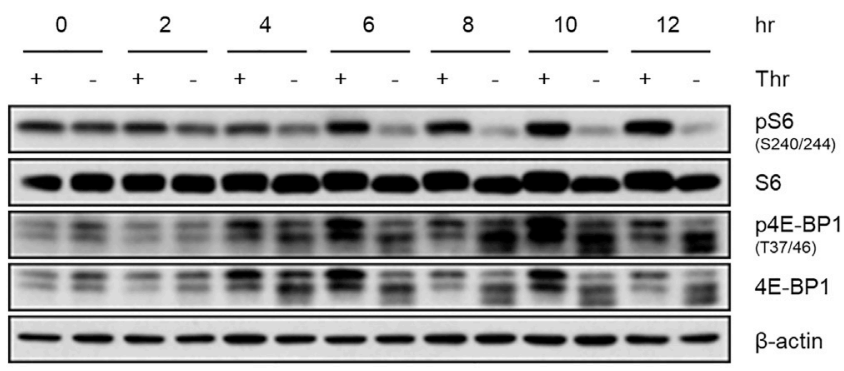

D
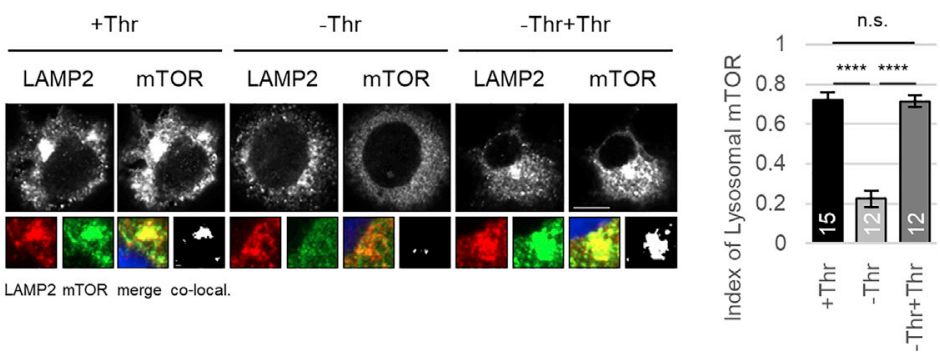

G

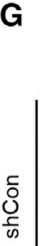

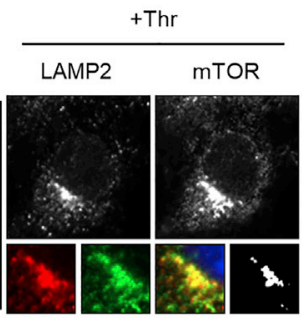

LAMP2 MTOR merge co-local.

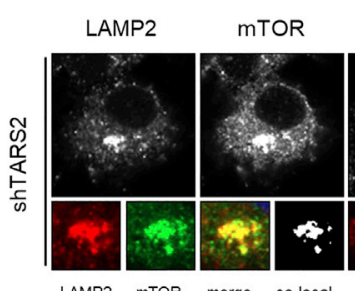

LAMP2 MTOR merge co-local

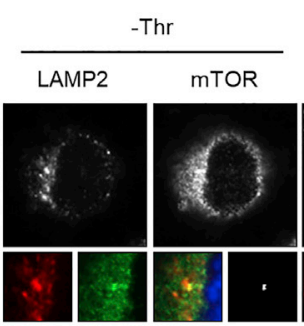

LAMP2

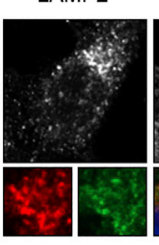

$\mathrm{hr} \quad \mathbf{F}$

F $\quad+$ Thr

$\frac{- \text { Thr }+ \text { Thr }}{+\frac{5}{+} \frac{10}{+} \frac{15}{+} \frac{30}{+} \frac{60}{+}}$

$\min$

shTARS2

TARS2

pS6

(S240/244)

S6

a-tubulin

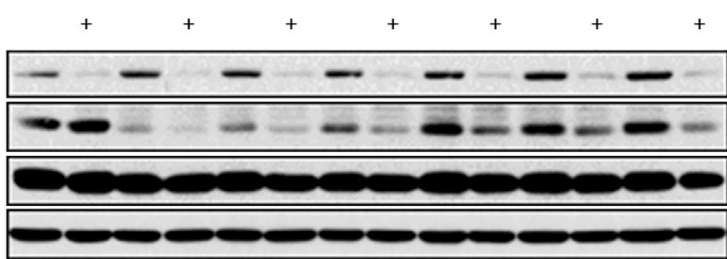

ShTARS2

TARS2

pS6

( $(5240 / 244)$

56

$\beta$-actin 


\section{$\infty$ CellPress}

\section{Molecular Cell Short Article}

et al., 2019; https://humancellmap.org). Through the exploration of the HumanCellMap database for ARSs, we noted the expected and near-exclusive recovery of mt-ARSs with $\mathrm{mt}$ baits. However, TARS2 was also readily recovered as a proximity interactor for the sole subunit of the Ragulator complex in the dataset (LAMTOR1) (Figure 1B). Except for TARS2 and $\mathrm{mt}$ isoleucyltRNA synthetase 2 (IARS2), which was also recovered as prey with LAMTOR1, albeit at a comparatively lower level than TARS2, no other mt-ARSs was significantly captured as prey with LAMTOR1 (Figure 1B). Consistently, LamTOR1 co-localized with TARS2 and IARS2, but not with mt-LARS2 (Figure 1C). This intriguing and surprising finding prompted us to explore the physical association of mt-ARSs with the lysosomal mTORC1 machinery. In contrast to IARS2, which is reportedly involved in

Q9 lung cancer tumorigenesis through the AKT/mTOR pathway (Di et al., 2019), the role of TARS2 in the mTORC1 pathway has remained unexplored. Our co-immunoprecipitation (colP) assay revealed that TARS2, but not TARS, interacted not only with Ragulator (LamTOR1-LamTOR5) but also with other mTOR complex components (mLST8, TEL2, and TTI1) and Rags (RagA-RagD) (Figure S1A). As expected, TARS interacted with its known binding partner 4EHP (Chapat et al., 2017; Jeong et al., 2019) (Figure S1A).

TARS2 mainly localizes to mitochondria because of its $\mathrm{N}$-terminal mitochondrial targeting sequence (MTS) (Figures S1B-S1D). Consistently, TARS2 was detected in the fraction representing a mixture of mitochondria and lysosomes (Figure 1D, mito. + lyso.), but not in the pure lysosomes (Figure 1D, lyso.), where the aa-sensing machinery, such as RagC and LamTOR4, resides. Nonetheless, a fraction of TARS2 proteins (10\% to $\sim 15 \%$ ) was present in the cytoplasm (Figure 1D, cyto.), implying the possibility of transient association of TARS2 with aa-sensing machinery at the cytoplasmic interface of lysosomes.

\section{Thr Modulates mTORC1 Activity through TARS2}

LARS functions as a Leu sensor in the mTORC1 pathway (Han et al., 2012), in addition to its canonical enzymatic charging of Leu to its cognate tRNA. Bearing this in mind, we examined the potential role of TARS2 in the Thr-mediated regulation of mTORC1. mTORC1 activity, indicated by phosphorylation of the ribosomal protein S6 (pS6 S240/244) and 4E-BP1 (p4E-
BP1 T37/46), was rapidly decreased by depletion of aas or Leu within $1 \mathrm{~h}$ ( $75 \%$ or $60 \%$ reduction, respectively) (Figure $2 \mathrm{~A}$ ). Whereas the effect was less drastic for the depletion of Thr alone for the same duration (30\% reduction), prolonged Thr starvation (12 h) dramatically reduced mTORC1 activity (85\% reduction) (Figure 2A). The low potency of Thr in the regulation of mTORC1 activity is likely because of the low cytoplasmic amount of TARS2 (Novoa et al., 2014) and lower binding affinity of TARS2 to Thr compared with cellular Thr concentration (Wang et al., 2016; Abu-Remaileh et al., 2017). Mechanistic target of rapamycin complex 2 (mTORC2) activity (pAKT S473) and TARS2 protein levels were not significantly altered by Thr starvation (Figure S2A). Reduced mTORC1 activity because of Thr starvation was reversed upon incubation with $800 \mu \mathrm{M}$ Thr (Figures 2C and S2B). Moreover, adding Thr at a low concentration $(8 \mu \mathrm{M})$ to Thr-starved cells was sufficient for the full recovery of mTORC1 activity within $90 \mathrm{~min}$ (Figure S2C). Notably, stimulating cells by serine or valine, two amino acids similar to Thr, failed to rescue mTORC1 activity (Figure S2C). mTORC1 is recruited to the lysosomal surface by Rags in response to aas (Sancak et al., 2010). Consistently, the lysosomal localization of mTOR, indicated by co-localization with LAMP2, was enhanced upon addition of Thr to Thr-starved cells (Figure 2D), demonstrating that Thr effectively controls the activity and lysosomal localization of mTORC1.

We next examined whether TARS2 is required for this process. Compared with control cells (control short hairpin RNA [shCon]), mTORC1 activity in TARS2 knockdown cells (TARS2 short hairpin RNA [shTARS2]) was further reduced by $12 \mathrm{~h}$ of Thr starvation (64\% and $85 \%$ reduction, respectively) (Figures $2 \mathrm{E}$ and S2D). Moreover, reduced mTORC1 activity was fully recovered within $15 \mathrm{~min}$ in shCon, but not in shTARS2, even 60 min after Thr stimulation (Figures $2 \mathrm{~F}$ and S2E), indicating that TARS2 plays a key role in the Thr-dependent regulation of $\mathrm{mTORC1}$ activity. These effects were independent of short hairpin RNA (shRNA) sequences (Figure S2F). Furthermore, the lysosomal recruitment of mTOR by Thr was abolished in shTARS2 (Figure $2 \mathrm{G}$ ), demonstrating that TARS2 is required for the Thr-mediated activation and lysosomal recruitment of mTORC1. As expected, mTORC1 activity in TARS knockdown cells in the presence/absence of Thr was almost identical to that in shCon (Figure S2G).

Figure 2. mTORC1 Activity and Localization Are Modulated by Thr in a TARS2-Dependent Manner

(A) mTORC1 activity after depletion of amino acids (aas), leucine (Leu), or threonine (Thr). HEK293T cells were starved for 1 or 12 h. pS6 S240/244 and p4E-BP1 T37/46 were used as mTORC1 activity markers.

(B) mTORC1 activity under starved (-) versus normal (+) Thr levels.

(C) Recovery of mTORC1 activity by Thr stimulation. HEK293T cells were starved of Thr for $15 \mathrm{~h}$ ( - Thr), followed by the addition of $800 \mu \mathrm{M}$ Thr ( - Thr+Thr). (D) mTOR lysosomal localization under starvation and supplementation of Thr. (Left) HEK293T cells were subjected to the same treatment as described in (C) but stimulated by Thr for $1 \mathrm{~h}$. Co-localization of LAMP2 and mTOR (co-local.), indicated by yellow spots in the merged image, is shown as white. Nuclei were stained by Hoechst (blue). Scale bar, $10 \mu \mathrm{m}$. (Right) Mean \pm SEM of the index of lysosomal mTOR, defined as the area of co-local. over the area of LAMP2, from the indicated number of images containing at least one cell is shown. n.s., not significant; ${ }^{\star \star \star *} p<0.0001$ (one-way ANOVA followed by Tukey's post hoc test). (E) mTORC1 activity in TARS2 knockdown cells under Thr starvation. HEK293T cells stably expressing control shRNA (shCon) or TARS2 shRNA (shTARS2) were used.

(F) Recovery of mTORC1 activity in shTARS2 by Thr stimulation. Cells were starved of Thr for $15 \mathrm{~h}$ before treating with $800 \mu \mathrm{M}$ Thr.

(G) mTOR lysosomal localization in TARS2 knockdown cells under starvation and stimulation of Thr. (Left) Cells were subjected to the same treatment as described in (D). Nuclei were stained by Hoechst (blue). Scale bar $10 \mu \mathrm{m}$. (Right) The index of lysosomal mTOR was analyzed as described in (D). n.s., not significant; ${ }^{\star} p<0.05 ;{ }^{\star \star \star *} \mathrm{p}<0.0001$ (two-way ANOVA followed by Sidak's post hoc test).

See also Figure S2. 
A

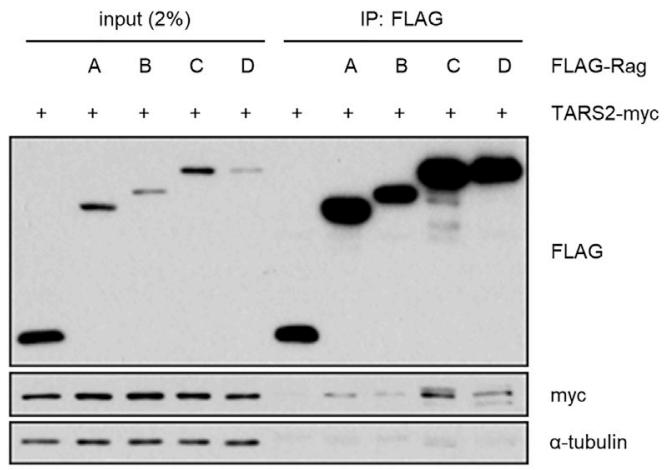

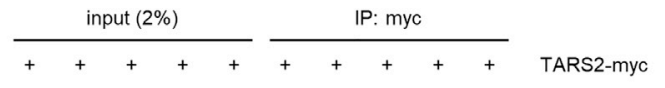

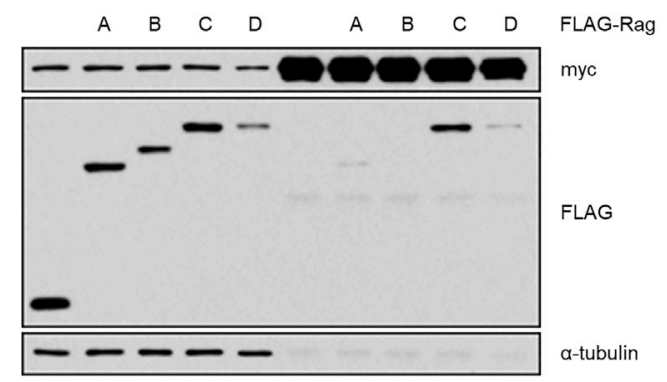

B

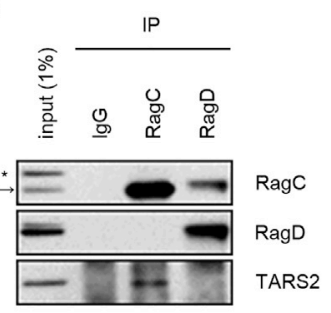

C

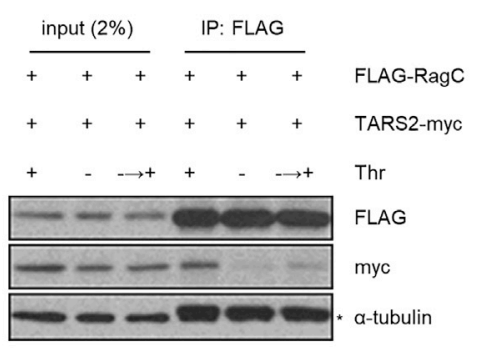

E

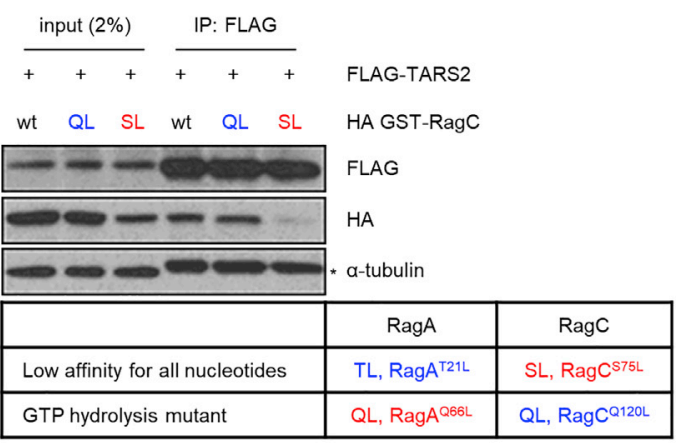

G

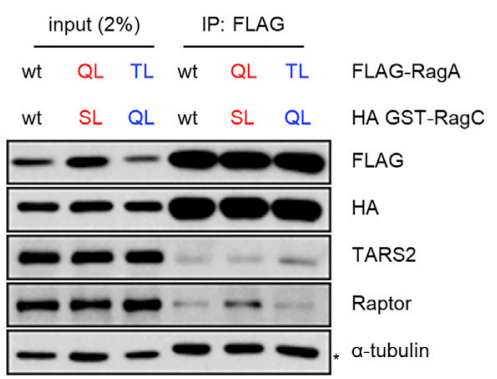

Figure 3. TARS2 Binds RagC in a Thr-Dependent and Nucleotide-Dependent Manner

(A) Preferential interaction of TARS2 with RagC. HEK293T cell lysates expressing the indicated plasmids were used for immunoprecipitation (IP) with an antiFLAG (left) or anti-myc (right) antibody.

(B) Interaction of endogenous TARS2 and RagC. HEK293T cell lysates were used for IP with an anti-RagC or anti-RagD antibody. Normal immunoglobulin G (IgG) was used as a negative control. An arrow and an asterisk indicate RagC and a non-specific band, respectively.

(C) Interaction of TARS2 with RagC in a Thr-dependent manner. HEK293T cells expressing RagC and TARS2 were starved of Thr for $15 \mathrm{~h}(-)$ and then treated with $800 \mu \mathrm{M}$ Thr for $1 \mathrm{~h}(-\rightarrow+)$. IP was performed using an anti-FLAG antibody. An asterisk indicates the heavy IgG chain.

(D) Interaction of mutant TARS2 with RagC. HEK293T cells were transfected with wild-type (WT), aminoacylation-defective (C409A and R438A), or editingdefective (H133A.H137A) TARS2 and RagC. IP was performed using an anti-hemagglutinin (HA) antibody. An asterisk indicates the heavy IgG chain. Empty vector, EV. 


\section{$\infty$ CellPress}

\section{Molecular Cell

GCN2 senses aa deficiency by responding to uncharged tRNA levels and inhibits mTORC1 activity through elF2 $\alpha$-ATF4-Sestrin2 (Ye et al., 2015). However, neither phosphorylation of elF2 $\alpha$ (pelF2 $\alpha$ S51), a marker of GCN2 activity triggered by aa deprivation, nor mRNA and protein levels of ATF4 and Sestrin2 were significantly altered between shCon and shTARS2 (Figure $\mathrm{S} 2 \mathrm{H}$ ), indicating that TARS2 plays a part in regulating mTORC1 activity in response to Thr levels independent of the elF2 $\alpha$-ATF4-Sestrin2 pathway.

\section{TARS2 Interacts with Rags}

Rags are essential for the aa-dependent mTORC1 activation by recruiting $\mathrm{mTORC} 1$ to the lysosomal surface (Sancak et al., 2008). We observed the interaction and co-localization of TARS2 with Rags, most preferentially with RagC (Figures 3A, S3A, and S3B). colP using TARS2 truncation mutants (Figure S3C, upper panel) showed that although TARS2 ${ }^{1-60}$ and TARS2 ${ }^{1-124}$ were not expressed, probably because of their inherent instability, RagC interacted with TARS2 ${ }^{1-399}$ (Figure S3C, lower panel, lane 10), but not with TARS2 ${ }^{1-300}$ (lane 5). This result suggested that TARS2 ${ }^{301-399}$, the intermediate region between the editing domain and the catalytic core domain $_{\mathbb{2}}$ is required for the interaction with RagC. TARS2 ${ }^{301-399}$ contains a segment (TARS2 ${ }^{382-398}$ ) that is unique to TARS2 of higher organisms (Figure S3D, upper panel), but deletion of this segment did not affect the interaction of TARS2 with RagC (Figure S3D, lower panel). Likewise, colP using RagC truncation mutants (Figure S3E, upper panel) showed that TARS2 interacts with RagC ${ }^{1-369}$ (Figure S3E, lower panel, lane 7), but not with $\mathrm{RagC}^{1-242}$ (lane 5). This result indicated that $\mathrm{RagC}^{243-369}$ is required for the interaction with TARS2. RagD displayed no interaction with TARS2 (lane 9), which is consistent with our endogenous colP data (Figure $3 \mathrm{~B}$ ). RagC $^{243-369}$ is mainly identical to the corresponding region of RagD ${ }^{244-370}$ (only 9 of 127 aas are different). However, single aa substitution mutants of RagC (Figure S3F, upper panel) did not abolish its interaction with TARS2 (Figure S3F, lower panel), likely indicating that $\geq 2$ aas in RagC $^{243-369}$ are required for this interaction. Consistently, in contrast to RagD knockdown, RagC knockdown cells could not recover mTORC1 activity by Thr stimulation (Figure S3G), demonstrating that TARS2 functions in the mTORC1 pathway through the preferential interaction with RagC.

The interaction between TARS2 and RagC was Thr dependent (Figure 3C). Among aminoacylation-defective (C409A and $\mathrm{R} 438 \mathrm{~A})$ and editing-defective $(\mathrm{H} 133 \mathrm{~A} \cdot \mathrm{H} 137 \mathrm{~A})$ TARS2 mutants (Chen et al., 2018), only the C409A mutant lost the interaction with RagC (Figure 3D). C409 coordinates a $\mathrm{Zn}^{2+}$ ion, which binds the $\alpha$-amino group and side-chain hydroxyl group of Thr, for recognition and discrimination of Thr at the active site, whereas
R438 interacts with the carboxyl group of Thr, $3^{\prime}$-CCA of tRNA, and $\alpha$-phosphate of ATP (Sankaranarayanan et al., 1999; Torres-Larios et al., 2003). Even though both TARS2 mutations are aminoacylation defective, previous studies using bacterial orthologs suggest that C409A is likely defective in Thr binding (Nureki et al., 1993; Sankaranarayanan et al., 1999), and R438A is likely defective in tRNA charging (Minajigi and Francklyn, 2008). Collectively, these results imply that TARS2 must be correctly charged with Thr to interact with RagC. We next examined whether the nucleotide state of RagC influences the interaction with TARS2. Intriguingly, TARS2 preferentially interacted with the GTP hydrolysis-defective RagC mutant (QL, RagC ${ }^{\text {Q120L }}$ ) mimicking GTP-RagC (Figure 3E). As expected, TARS did not bind RagC, regardless of the nucleotide state (Figure 3F). Furthermore, TARS2 preferentially interacted with Rags composed of GDP-RagA (TL) and GTP-RagC (QL) (Figure 3G). Collectively, these data demonstrate that TARS2 binds GTPRagC in the presence of Thr.

\section{TARS2 Induces GTP Loading of RagA in Response to Thr Stimulation}

To examine the role of TARS2 in aa signaling and the regulation of mTORC1 activity, we explored the nucleotide state of Rags in the presence/absence of Thr. In parallel with mTORC1 activity (pS6), GTP loading of RagA was most dramatically altered by Thr levels (Figure S4A). Although Thr starvation reduced GTP loading of RagA similarly between shCon and shTARS2, Thr stimulation did not elevate GTP loading of RagA in shTARS2 (Figure 4A). Because GTP-RagA is sufficient to activate mTORC1 (Kim et al., 2008), these results demonstrate that TARS2 is required for the activation of $\mathrm{mTORC} 1$ upon replenishing Thr.

The signals emanating from intracellular aa levels are transmitted to Rags through two distinct pathways. Cytoplasmic aa sensors such as LARS (Han et al., 2012), Sestrin2 (Wolfson et al., 2016), and CASTOR (Chantranupong et al., 2016) detect free aas, and the lysosomal aa sensors such as v-ATPase-Ragulator (Bar-Peled et al., 2012) and SLC38A9 (Rebsamen et al., 2015) detect lysosomal efflux of aas. To determine the mechanism by which TARS2 senses Thr levels, we pre-treated cells with the v-ATPase inhibitor bafilomycin A1 (BafA1) to block lysosomal Thr efflux (Abu-Remaileh et al., 2017). Recovery of mTORC1 activity by Thr replenishment was similar between shCon and shTARS2 with or without BafA1 (Figure S4B), indicating that TARS2 detects cytoplasmic free Thr levels.

Because the nucleotide exchange on guanosine triphosphatases (GTPases) is achieved by GEFs, we compared the effect of TARS2 on GTP loading of RagA with two known GEFs for Rags: Ragulator (Bar-Peled et al., 2012) and SLC38A9 (Shen et al., 2018). Although GTP loading of RagA was increased upon Thr Q10

(E) Preferential interaction of TARS2 with GTP-RagC. HEK293T cells expressing TARS2 with WT, GTP- (QL), or GDP- (SL) RagC were subjected to IP using an anti-FLAG antibody. An asterisk indicates the heavy IgG chain.

(F) Interaction of TARS2, but not TARS, with RagC. HEK293T cells expressing WT, GTP- (QL), or GDP- (SL) RagC with TARS or TARS2 were subjected to IP using an anti-HA antibody.

(G) Preferential interaction of TARS2 with inactive Rags. HEK293T cells expressing WT, GTP - (QL), or GDP - (TL) RagA and WT, GDP - (SL), or GTP - (QL) RagC were subjected to IP using an anti-FLAG antibody. An asterisk indicates the heavy IgG chain.

See also Figure S3. 
A

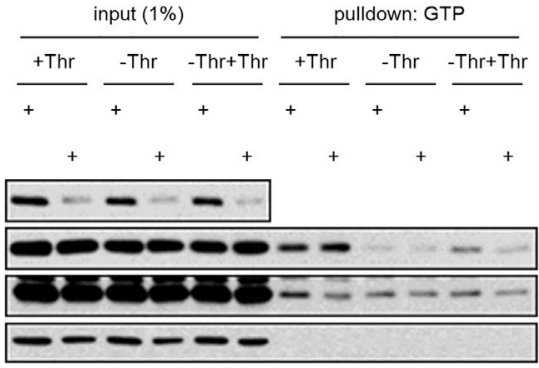

C

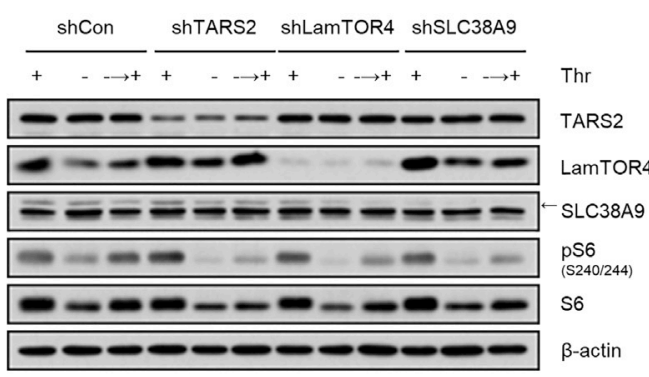

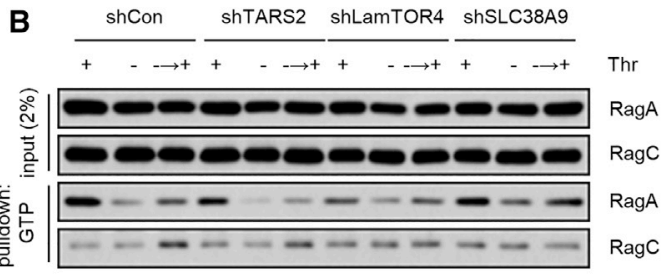

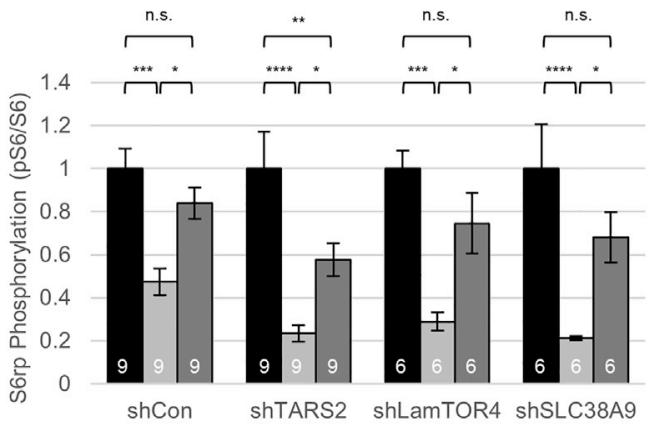

- + Thr $\quad$ 口-Thr 口-Thr+Thr
D

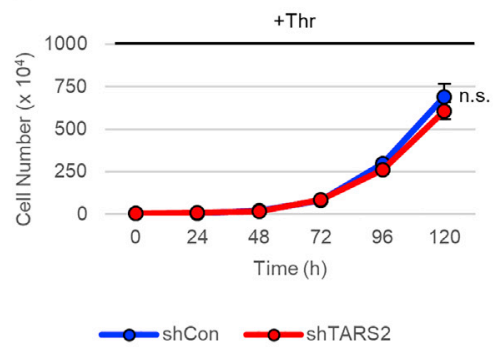

$\mathbf{F}$

Figure 4. TARS2 Activates mTORC1 in Response to Thr by Increasing GTP Loading of RagA

(A) GTP loading of Rags in TARS2 knockdown cells under starvation and stimulation of Thr. Cells were starved of Thr for $15 \mathrm{~h}$ (-Thr) and then stimulated by $800 \mu \mathrm{M}$ Thr for $1 \mathrm{~h}(-\mathrm{Thr}+\mathrm{Thr})$. Cells were subjected to GTP-agarose pull-down assay. An asterisk indicates a non-specific band.

(B) GTP loading of Rags in cells lacking TARS2 or GEF under starvation and stimulation of Thr. Cells stably expressing the indicated shRNA were subjected to the same treatment and GTP-agarose pull-down assay as described in (A).

(C) Recovery of mTORC1 activity in cells lacking TARS2 or GEF under starvation and stimulation of Thr. (Left) Cells were subjected to the same treatment as described in (A). An arrow indicates SLC38A9. (Right) Mean \pm SEM of pS6 from 6 to 9 independent blots is shown. n.s., not significant; ${ }^{*} p<0.05 ;{ }^{* *} p<0.01$; ${ }^{* * *} p<0.001 ;{ }^{* * \star *} p<0.0001$ (two-way ANOVA followed by Tukey's post hoc test). 


\section{$\infty$ CellPress}

stimulation in cells lacking LamTOR4 (shLamTOR4) or SLC38A9 (shSLC38A9) similar to shCon, GTP loading of RagA in shTARS2 was not increased to the same levels (Figure 4B). Consistently, recovery of mTORC1 activity by Thr stimulation in shTARS2 was $30 \%$ lower than in shCon, whereas the recovery in shLamTOR4 or shSLC38A9 was comparable to that in shCon (Figure $4 \mathrm{C}$ ). Because it lacks known functional GEF domains, TARS2 is not likely a potential GEF, but it could act as a potent regulator of Thr-sensitive GTP loading of RagA and mTORC1 activity, presumably by modulating the activity of a yet-unknown GEF.

TARS2 Controls Cell Proliferation and Global mRNA Translation Efficiency by Activating mTORC1

Finally, we examined the physiological impact of TARS2-mediated mTORC1 activation by Thr. Cell proliferation was almost identical between shCon and shTARS2 under normal Thr levels (Figure 4D, left), probably because of the maintenance of basal mTORC1 activity (Figures 2E and S2D). However, although the proliferation of both cells was similarly suppressed under Thr depletion (Figure 4D, right, dotted lines), recovery of the proliferation rate in shTARS2 upon Thr replenishment was 25\% lower than the rate in shCon (Figure 4D, right, solid lines). We also assessed global mRNA translation, which is controlled by mTORC1, via polysome profiling (Gandin et al., 2014). The polysome profiles of shCon and shTARS2 appeared identical under normal (Figure S4C) and Thr-free conditions (Figure S4D, lightcolored curves). However, recovery of the ratio of polysomes to monosomes, an indicator of translation efficiency, by Thr supplementation in shTARS2 was $25 \%$ lower than in shCon (Figures 4E and S4D, dark-colored curves), demonstrating an important physiological role of TARS2 in the activation of mTORC1 by Thr stimulation.

In conclusion, we discovered a mechanism that controls the lysosomal localization and activity of mTORC1 in response to Thr levels through TARS2. We demonstrated that the interaction of TARS2 with components of the aa-sensing machinery, which impinges on mTORC1, plays a crucial role in Thr-mediated activation of mTORC1. We further revealed that in the presence of Thr, TARS2 binds GTP-RagC and promotes the recruitment of mTORC1 by increasing GTP loading of RagA (Figure 4F). Collectively, these findings present the specific regulation of mTORC1 activity by aas through a mt protein and are a further example of the expanding role of $\mathrm{mt}$ proteins in various cellular compartments.

\section{STAR $\star$ METHODS}

Detailed methods are provided in the online version of this paper and include the following:
- KEY RESOURCES TABLE

- RESOURCE AVAILABILITY

Lead Contact

O Materials Availability

- Data and Code Availability

- METHOD DETAILS

O Cell culture, amino acid starvation, stimulation, and transient transfection

o Lentivirus production

O Immunoprecipitation

O Subcellular fractionation

O Immunofluorescence

- Proximity ligation assay (PLA)

$\bigcirc$ GTP-agarose pulldown assay

Polysome profiling

\section{SUPPLEMENTAL INFORMATION}

Supplemental Information can be found online at https://doi.org/10.1016/j. molcel.2020.11.036.

\section{ACKNOWLEDGMENTS}

We thank Devon Merkley for technical assistance and Gyan Prakash for constructive discussion. This work was supported by the Canadian Institutes of Health Research (ClHR) Foundation grants FDN-148423 (to N.S.) and FDN143301 (to A.-C.G.). S.-H.K. was supported by the Basic Science Research Program through the National Research Foundation of Korea (NRF) funded by the Ministry of Education (2019R1A6A3A03034060) and is supported by the Canderel Postdoctoral Fellowship. P.W. is supported by the Fonds de la recherche en sante du Quebec (FRQS) Postdoctoral Fellowship. C.D.G. is supported by a Frederick Banting and Charles Best Canada Graduate Scholarship. G.G.H. was supported by a Parkinson Canada Basic Research Fellowship. A.-C.G. is the Canada Research Chair in Functional Proteomics and the Lea Reichmann Chair in Cancer Proteomics. S.M.J. is supported by the Patrick Johnston Research Fellowship at Queen's University Belfast and the Q13 UK Medical Research Council Confidence in Concept Scheme, Graphical abstract and Figure $4 \mathrm{~F}$ were created with BioRender.com.

\section{AUTHOR CONTRIBUTIONS}

S.-H.K. designed and performed experiments, analyzed data, and wrote the manuscript; J.-H.C. and P.W. performed experiments and edited the manuscript; C.D.G. and G.G.H. performed the BiolD assay and provided the HumanCellMap dataset; A.-C.G. designed and analyzed the BiolD data and edited the manuscript; S.M.J. contributed to the conception of the project, designed experiments, supervised the project, and wrote the manuscript; and N.S. supervised the project, analyzed data, edited the manuscript, and secured funding.

\section{DECLARATION OF INTERESTS}

The authors declare no competing interests.

(D) Proliferation of TARS2 knockdown cells under starvation and stimulation of Thr. (Left) Proliferation curves of shCon (blue) or shTARS2 (red) cells in a complete medium (+Thr) are shown. (Right) Proliferation curves of shCon (blue) or shTARS2 (red) cells in a Thr-free medium (-Thr, dotted line) are shown. $36 \mathrm{~h}$ after seeding, cells were supplemented with $800 \mu \mathrm{M}$ Thr (-Thr+Thr, solid line). The curves show mean \pm SEM of 3 independent replicates. n.s., not significant; * $p<0.05$ (twoway ANOVA followed by Sidak's post hoc test).

(E) Global mRNA translation in TARS2 knockdown cells under starvation and stimulation of Thr. Mean \pm SEM of the ratio of polysomes to monosomes from 3 independent polysome profiles. n.s., not significant; ${ }^{* \star} \mathrm{p}<0.01$ (two-way ANOVA followed by Sidak's post hoc test).

(F) Model of TARS2-mediated mTORC1 activation by Thr stimulation. (1) GDP-RagA/GTP-RagC forms an inactive complex under Thr-deprived condition. (2) Thr stimulation increases charging of TARS2 with Thr. (3) Thr-charged TARS2 is recruited to GTP-RagC. (4) GTP loaded on RagC is hydrolyzed (by an unknown mechanism) and TARS2 recruits a GEF for RagA. (5) GEF replaces GDP on RagA with GTP. Rags then become active, which is sufficient to recruit mTORC1 by directly binding with $_{2}$ Raptor. See also Figure S4. 


\section{Molecular Cell Short Article}

Received: November 18, 2019

Revised: May 14, 2020

Accepted: November 19, 2020

Published: December 18, 2020

\section{REFERENCES}

Abu-Remaileh, M., Wyant, G.A., Kim, C., Laqtom, N.N., Abbasi, M., Chan, S.H., Freinkman, E., and Sabatini, D.M. (2017). Lysosomal metabolomics reveals V-ATPase- and mTOR-dependent regulation of amino acid efflux from lysosomes. Science 358, 807-813.

Arif, A., Terenzi, F., Potdar, A.A., Jia, J., Sacks, J., China, A., Halawani, D. Vasu, K., Li, X., Brown, J.M., et al. (2017). EPRS is a critical mTORC1-S6K1 effector that influences adiposity in mice. Nature 542, 357-361.

Bar-Peled, L., Schweitzer, L.D., Zoncu, R., and Sabatini, D.M. (2012). Ragulator is a GEF for the rag GTPases that signal amino acid levels to mTORC1. Cell 150, 1196-1208.

Bar-Peled, L., Chantranupong, L., Cherniack, A.D., Chen, W.W., Ottina, K.A. Grabiner, B.C., Spear, E.D., Carter, S.L., Meyerson, M., and Sabatini, D.M. (2013). A Tumor suppressor complex with GAP activity for the Rag GTPases that signal amino acid sufficiency to mTORC1. Science 340, 1100-1106.

Ben-Sahra, I., and Manning, B.D. (2017). mTORC1 signaling and the metabolic control of cell growth. Curr. Opin. Cell Biol. 45, 72-82.

Bonfils, G., Jaquenoud, M., Bontron, S., Ostrowicz, C., Ungermann, C., and De Virgilio, C. (2012). Leucyl-tRNA synthetase controls TORC1 via the EGO complex. Mol. Cell 46, 105-110.

Chantranupong, L., Scaria, S.M., Saxton, R.A., Gygi, M.P., Shen, K., Wyant, G.A., Wang, T., Harper, J.W., Gygi, S.P., and Sabatini, D.M. (2016). The CASTOR proteins are arginine sensors for the mTORC1 pathway. Cell 165, 153-164.

Chapat, C., Jafarnejad, S.M., Matta-Camacho, E., Hesketh, G.G., Gelbart, I.A., Attig, J., Gkogkas, C.G., Alain, T., Stern-Ginossar, N., Fabian, M.R., et al. (2017). Cap-binding protein 4EHP effects translation silencing by microRNAs. Proc. Natl. Acad. Sci. USA 114, 5425-5430.

Chen, Y., Ruan, Z.R., Wang, Y., Huang, Q., Xue, M.Q., Zhou, X.L., and Wang, E.D. (2018). A threonyl-tRNA synthetase-like protein has tRNA aminoacylation and editing activities. Nucleic Acids Res. 46, 3643-3656.

Dai, W.T., White, R.R., Liu, J.X., and Liu, H.Y. (2018). Seryl-tRNA synthetasemediated essential amino acids regulate $\beta$-casein synthesis via cell proliferation and mammalian target of rapamycin (mTOR) signaling pathway in bovine mammary epithelial cells. J. Dairy Sci. 101, 10456-10468.

Di, X., Jin, X., Ma, H., Wang, R., Cong, S., Tian, C., Liu, J., Zhao, M., Li, R., and Wang, K. (2019). The oncogene IARS2 promotes non-small cell lung cancer tumorigenesis by activating the AKT/mTOR pathway. Front. Oncol. 9, 393.

Dibble, C.C., and Cantley, L.C. (2015). Regulation of mTORC1 by PI3K signaling. Trends Cell Biol. 25, 545-555.

Efeyan, A., Zoncu, R., and Sabatini, D.M. (2012). Amino acids and mTORC1: from lysosomes to disease. Trends Mol. Med. 18, 524-533.

Gandin, V., Sikström, K., Alain, T., Morita, M., McLaughlan, S., Larsson, O., and Topisirovic, I. (2014). Polysome fractionation and analysis of mammalian translatomes on a genome-wide scale. J. Vis. Exp. 87, 51455.

Go, C.D., Knight, J.D.R., Rajasekharan, A., Rathod, B., Hesketh, G.G., Abe, K.T., Youn, J.Y., Samavarchi-Tehrani, P., Zhang, H., Zhu, L.Y., et al. (2019). A proximity biotinylation map of a human cell. bioRxiv. https://doi.org/10. 1101/796391.

Gu, X., Orozco, J.M., Saxton, R.A., Condon, K.J., Liu, G.Y., Krawczyk, P.A., Scaria, S.M., Harper, J.W., Gygi, S.P., and Sabatini, D.M. (2017). SAMTOR is an S-adenosylmethionine sensor for the mTORC1 pathway. Science 358, 813-818.

Gupta, G.D., Coyaud, É., Gonçalves, J., Mojarad, B.A., Liu, Y., Wu, Q., Gheiratmand, L., Comartin, D., Tkach, J.M., Cheung, S.W., et al. (2015). A dynamic protein interaction landscape of the human centrosome-cilium interface. Cell 163, 1484-1499.
Han, J.M., Jeong, S.J., Park, M.C., Kim, G., Kwon, N.H., Kim, H.K., Ha, S.H., Ryu, S.H., and Kim, S. (2012). Leucyl-tRNA synthetase is an intracellular leucine sensor for the mTORC1-signaling pathway. Cell 149, 410-424.

Hara, K., Yonezawa, K., Weng, Q.P., Kozlowski, M.T., Belham, C., and Avruch, J. (1998). Amino acid sufficiency and mTOR regulate p70 S6 kinase and elF-4E BP1 through a common effector mechanism. J. Biol. Chem. 273, 14484-14494.

Hay, N., and Sonenberg, N. (2004). Upstream and downstream of mTOR. Genes Dev. 18, 1926-1945.

He, X.D., Gong, W., Zhang, J.N., Nie, J., Yao, C.F., Guo, F.S., Lin, Y., Wu, X.H., $\mathrm{Li}, \mathrm{F}$., Li, J., et al. (2018). Sensing and transmitting intracellular amino acid signals through reversible lysine aminoacylations. Cell Metab. 27, 151-166.e6.

Jeong, S.J., Park, S., Nguyen, L.T., Hwang, J., Lee, E.Y., Giong, H.K., Lee, J.S., Yoon, I., Lee, J.H., Kim, J.H., et al. (2019). A threonyl-tRNA synthetasemediated translation initiation machinery. Nat. Commun. 10, 1357.

Jewell, J.L., Kim, Y.C., Russell, R.C., Yu, F.X., Park, H.W., Plouffe, S.W., Tagliabracci, V.S., and Guan, K.L. (2015). Metabolism. Differential regulation of mTORC1 by leucine and glutamine. Science 347, 194-198.

Jung, J.W., Macalino, S.J.Y., Cui, M., Kim, J.E., Kim, H.J., Song, D.G., Nam, S.H., Kim, S., Choi, S., and Lee, J.W. (2019). Transmembrane $4 \mathrm{~L}$ six family member 5 senses arginine for mTORC1 signaling. Cell Metab. 29, 13061319.e7.

Kim, D.H., Sarbassov, D.D., Ali, S.M., King, J.E., Latek, R.R., ErdjumentBromage, H., Tempst, P., and Sabatini, D.M. (2002). mTOR interacts with raptor to form a nutrient-sensitive complex that signals to the cell growth machinery. Cell 110, 163-175.

Kim, E., Goraksha-Hicks, P., Li, L., Neufeld, T.P., and Guan, K.L. (2008) Regulation of TORC1 by Rag GTPases in nutrient response. Nat. Cell Biol. 10, 935-945.

Luo, C., Qi, H., Huang, X., Li, M., Zhang, L., Lin, Y., and Gao, X. (2019). GlyRS is a new mediator of amino acid-induced milk synthesis in bovine mammary epithelial cells. J. Cell. Physiol. 234, 2973-2983.

Minajigi, A., and Francklyn, C.S. (2008). RNA-assisted catalysis in a protein enzyme: The 2'-hydroxyl of tRNA(Thr) A76 promotes aminoacylation by threonyl-tRNA synthetase. Proc. Natl. Acad. Sci. USA 105, 17748-17753.

Novoa, E.M., Camacho, N., Tor, A., Wilkinson, B., Moss, S., Marín-García, P., Azcárate, I.G., Bautista, J.M., Mirando, A.C., Francklyn, C.S., et al. (2014). Analogs of natural aminoacyl-tRNA synthetase inhibitors clear malaria in vivo. Proc. Natl. Acad. Sci. USA 111, E5508-E5517.

Nureki, O., Kohno, T., Sakamoto, K., Miyazawa, T., and Yokoyama, S. (1993). Chemical modification and mutagenesis studies on zinc binding of aminoacyltRNA synthetases. J. Biol. Chem. 268, 15368-15373.

Rebsamen, M., Pochini, L., Stasyk, T., de Araújo, M.E.G., Galluccio, M., Kandasamy, R.K., Snijder, B., Fauster, A., Rudashevskaya, E.L., Bruckner, M., et al. (2015). SLC38A9 is a component of the lysosomal amino acid sensing machinery that controls mTORC1. Nature 519, 477-481.

Sancak, Y., Peterson, T.R., Shaul, Y.D., Lindquist, R.A., Thoreen, C.C., BarPeled, L., and Sabatini, D.M. (2008). The Rag GTPases bind raptor and mediate amino acid signaling to mTORC1. Science 320, 1496-1501.

Sancak, Y., Bar-Peled, L., Zoncu, R., Markhard, A.L., Nada, S., and Sabatini, D.M. (2010). Ragulator-Rag complex targets mTORC1 to the lysosomal surface and is necessary for its activation by amino acids. Cell 141, 290-303.

Sankaranarayanan, R., Dock-Bregeon, A.C., Romby, P., Caillet, J., Springer, M., Rees, B., Ehresmann, C., Ehresmann, B., and Moras, D. (1999). The structure of threonyl-tRNA synthetase-tRNA $\left.{ }^{\text {Thr }}\right)$ complex enlightens its repressor activity and reveals an essential zinc ion in the active site. Cell 97, 371-381.

Saxton, R.A., and Sabatini, D.M. (2017). mTOR signaling in growth, metabolism, and disease. Cell 168, 960-976.

Sekiguchi, T., Hirose, E., Nakashima, N., li, M., and Nishimoto, T. (2001). Novel G proteins, Rag C and Rag D, interact with GTP-binding proteins, Rag A and Rag B. J. Biol. Chem. 276, 7246-7257. 


\section{$\infty$ CellPress}

\section{Molecular Cell

Shen, K., and Sabatini, D.M. (2018). Ragulator and SLC38A9 activate the Rag GTPases through noncanonical GEF mechanisms. Proc. Natl. Acad. Sci. USA 115, 9545-9550.

Stafa, K., Tsika, E., Moser, R., Musso, A., Glauser, L., Jones, A., Biskup, S., Xiong, Y., Bandopadhyay, R., Dawson, V.L., et al. (2014). Functional interaction of Parkinson's disease-associated LRRK2 with members of the dynamin GTPase superfamily. Hum. Mol. Genet. 23, 2055-2077.

Torres-Larios, A., Sankaranarayanan, R., Rees, B., Dock-Bregeon, A.C., and Moras, D. (2003). Conformational movements and cooperativity upon amino acid, ATP and tRNA binding in threonyl-tRNA synthetase. J. Mol. Biol. 331, 201-211.

Wang, S., Tsun, Z.Y., Wolfson, R.L., Shen, K., Wyant, G.A., Plovanich, M.E., Yuan, E.D., Jones, T.D., Chantranupong, L., Comb, W., et al. (2015). Metabolism. Lysosomal amino acid transporter SLC38A9 signals arginine sufficiency to mTORC1. Science 347, 188-194.

Wang, Y., Zhou, X.L., Ruan, Z.R., Liu, R.J., Eriani, G., and Wang, E.D. (2016). A human disease-causing point mutation in mitochondrial threonyl-tRNA syn- thetase induces both structural and functional defects. J. Biol. Chem. 291 6507-6520.

Wolfson, R.L., and Sabatini, D.M. (2017). The dawn of the age of amino acid sensors for the mTORC1 pathway. Cell Metab. 26, 301-309.

Wolfson, R.L., Chantranupong, L., Saxton, R.A., Shen, K., Scaria, S.M., Cantor, J.R., and Sabatini, D.M. (2016). Sestrin2 is a leucine sensor for the mTORC1 pathway. Science 351, 43-48.

Wullschleger, S., Loewith, R., and Hall, M.N. (2006). TOR signaling in growth and metabolism. Cell 124, 471-484.

Ye, J., Palm, W., Peng, M., King, B., Lindsten, T., Li, M.O., Koumenis, C., and Thompson, C.B. (2015). GCN2 sustains mTORC1 suppression upon amino acid deprivation by inducing Sestrin2. Genes Dev. 29, 2331-2336.

Youn, J.Y., Dunham, W.H., Hong, S.J., Knight, J.D.R., Bashkurov, M., Chen, G.I., Bagci, H., Rathod, B., MacLeod, G., Eng, S.W.M., et al. (2018). High-density proximity mapping reveals the subcellular organization of mRNA-associated granules and bodies. Mol. Cell 69, 517-532.e11. 
Molecular Cell

Short Article

\section{Q5 Q6 STAR $\star$ METHODS}

\section{KEY RESOURCES TABLE}

\begin{tabular}{|c|c|c|}
\hline REAGENT or RESOURCE & SOURCE & IDENTIFIER \\
\hline \multicolumn{3}{|l|}{ Antibodies } \\
\hline Rabbit 4E-BP1 & Cell Signaling & Cat\# 9644; RRID:AB_2097841 \\
\hline Rabbit Phospho-4E-BP1 Thr37/46 & Cell Signaling & Cat\# 9459; RRID:AB_330985 \\
\hline Rabbit AKT & Cell Signaling & Cat\# 4691; RRID:AB_915783 \\
\hline Rabbit Phosphor-AKT Ser473 & Cell Signaling & Cat\# 9271; RRID:AB_329825 \\
\hline Rabbit elF2 $\alpha$ & Cell Signaling & Cat\# 9722; RRID:AB_2230924 \\
\hline Normal rabbit lgG & Cell Signaling & Cat\# 2729; RRID:AB_1031062 \\
\hline Rabbit LamTOR4 & Cell Signaling & Cat\# 13140; RRID:AB_2798129 \\
\hline Rabbit mTOR & Cell Signaling & Cat\# 2983; RRID:AB_2105622 \\
\hline Rabbit Myc & Cell Signaling & Cat\# 2278; RRID:AB_490778 \\
\hline Rabbit RagA & Cell Signaling & Cat\# 4357; RRID:AB_10545136 \\
\hline Rabbit RagB & Cell Signaling & Cat\# 8150; RRID:AB_11178806 \\
\hline Rabbit Phospho-S6rp Ser240/244 & Cell Signaling & Cat\# 5364; RRID:AB_10694233 \\
\hline Rabbit S6rp & Cell Signaling & Cat\# 2217; RRID:AB_331355 \\
\hline Rabbit ATF4 & Abcam & Cat\# ab184909; RRID:AB_2819059 \\
\hline Mouse LAMP2 & Abcam & Cat\# ab25631; RRID:AB_470709 \\
\hline Mouse RagC & Abcam & Cat\# ab76577; RRID:AB_2301060 \\
\hline Rabbit SLC38A9 & Abcam & Cat\# ab81687; RRID:AB_1860451 \\
\hline Rabbit Sestrin2 & ProteinTech & Cat\# 10795-1-AP; RRID:AB_2185480 \\
\hline Rabbit TARS & ProteinTech & Cat\# 14773-1-AP; RRID:AB_2200654 \\
\hline Rabbit TARS2 & ProteinTech & Cat\# 15067-1-AP; RRID:AB_2200668 \\
\hline Rabbit IARS2 & ProteinTech & Cat\# 17170-1-AP; RRID:AB_2264448 \\
\hline Rabbit LARS2 & ProteinTech & Cat\# 17097-1-AP; RRID:AB_2132807 \\
\hline Mouse $\alpha$-tubulin & Santa Cruz Biotechnology & Cat\# sc-23948; RRID:AB_628410 \\
\hline Mouse TOM20 & Santa Cruz Biotechnology & Cat\# sc-17764; RRID:AB_628381 \\
\hline Mouse HA & Covance & Cat\# MMS-101R-1000; RRID:AB_291262 \\
\hline Mouse $\beta$-actin & Sigma & Cat\# A5441; RRID:AB_476744 \\
\hline Mouse FLAG & Sigma & Cat\# F3165; RRID:AB_259529 \\
\hline Rabbit HA & Sigma & Cat\# H6908; RRID:AB_260070 \\
\hline Rabbit Phospho-elF2 $\alpha$ Ser52 & Invitrogen & Cat\# 44-728G; RRID:AB_1500038 \\
\hline Mouse RagC & Novus Biologicals & Cat\# NBP2-32202 \\
\hline Mouse RagD & Novus Biologicals & Cat\# NBP2-32106 \\
\hline Goat c-Myc & Genetex & Cat\# GTX30518; RRID:AB_385719 \\
\hline Mouse Myc & BioShop & Cat\# TAG003.100 \\
\hline \multicolumn{3}{|c|}{ Chemicals, Peptides, and Recombinant Proteins } \\
\hline Puromycin & BioShop & Cat\# PUR333.500 \\
\hline Cycloheximide & BioShop & Cat\# CYC003 \\
\hline Bafilomycin A1 & Sigma & Cat\# B1793 \\
\hline \multicolumn{3}{|l|}{ Critical Commercial Assays } \\
\hline $\begin{array}{l}\text { Duolink In Situ Red Starter Kit Mouse/ } \\
\text { Rabbit }\end{array}$ & Sigma & Cat\# DUO92101 \\
\hline $\begin{array}{l}\text { QuikChange Lightning Site-Directed } \\
\text { Mutagenesis Kit }\end{array}$ & Agilent & Cat\# 210518 \\
\hline
\end{tabular}




\begin{tabular}{|c|c|c|}
\hline Continued & & \\
\hline REAGENT or RESOURCE & SOURCE & IDENTIFIER \\
\hline \multicolumn{3}{|l|}{ Deposited Data } \\
\hline Unprocessed data & This study & https://doi.org/10.17632/rr89y6c68m.1 \\
\hline \multicolumn{3}{|l|}{ Experimental Models: Cell Lines } \\
\hline HEK293T & ATCC & CRL-3216 \\
\hline HeLa & ATCC & CCL-2 \\
\hline \multicolumn{3}{|l|}{ Oligonucleotides } \\
\hline $\begin{array}{l}\text { Human Atf4 realtime PCR primer forward: } \\
\text { TCTACCAGGGGCTCTCCAAAT }\end{array}$ & This study & N/A \\
\hline $\begin{array}{l}\text { Human Atf4 realtime PCR primer reverse: } \\
\text { CCTAGTGGCTGCTGTCTTGTT }\end{array}$ & This study & N/A \\
\hline $\begin{array}{l}\text { Human Sestrin2 realtime PCR primer } \\
\text { forward: CCGCCATCAGTGTTCTTACCT }\end{array}$ & This study & N/A \\
\hline $\begin{array}{l}\text { Human Sestrin2 realtime PCR primer } \\
\text { reverse: CTGGATGTGTTCCTTGGTGATG }\end{array}$ & This study & N/A \\
\hline \multicolumn{3}{|l|}{ Recombinant DNA } \\
\hline pRK5 HA GST-RagC wt & Addgene & Cat\# 19304 \\
\hline pRK5 HA GST-RagC 75L & Addgene & Cat\# 19305 \\
\hline pRK5 HA GST-RagC 120L & Addgene & Cat\# 19306 \\
\hline pRK5 HA GST-RagC mutants & This study & $\mathrm{N} / \mathrm{A}$ \\
\hline pRK5 HA GST-RagD wt & This study & Uniprot: Q9NQL2-1 \\
\hline pcDNA4 TARS2-his/myc wt and mutants & This study & Uniprot: Q9BW92-1 \\
\hline pcDNA4 TARS-his/myc wt & This study & Uniprot: P26639-1 \\
\hline pcDNA5 3xFLAG-RagA wt and mutants & This study & Uniprot: Q7L523-1 \\
\hline pcDNA5 3xFLAG-RagC wt & This study & Uniprot: Q9HB90-1 \\
\hline TARS2 shRNA \#1 & Sigma & Cat\# TRCN0000045434 \\
\hline TARS2 shRNA \#2 & Sigma & Cat\# TRCN0000045436 \\
\hline TARS2 shRNA \#3 & Sigma & Cat\# TRCN0000045437 \\
\hline TARS shRNA \#1 & Sigma & Cat\# TRCN0000344636 \\
\hline TARS shRNA \#2 & Sigma & Cat\# TRCN0000333070 \\
\hline LamTOR4 shRNA & Sigma & Cat\# TRCN0000281609 \\
\hline SLC38A9 shRNA & Sigma & Cat\# TRCN0000150620 \\
\hline RagC shRNA & Sigma & Cat\# TRCN0000072874 \\
\hline RagD shRNA & Sigma & Cat\# TRCN0000059537 \\
\hline Control shRNA & Sigma & Cat\# SHC002 \\
\hline pLP1 & Invitrogen & Cat\# K497500 \\
\hline pLP2 & Invitrogen & Cat\# K497500 \\
\hline pLP/VSVG & Invitrogen & Cat\# K497500 \\
\hline \multicolumn{3}{|l|}{ Software and Algorithms } \\
\hline GraphPad Prism & GraphPad & https://www.graphpad.com \\
\hline ImageJ & National Institute of Health & https://imagej.nih.gov/ij/ \\
\hline Fiji & Fiji & https://image.net/fiji \\
\hline GIMP & The GIMP Team & https://www.gimp.org/ \\
\hline ZEN blue edition & Zeiss & $\begin{array}{l}\text { https://www.zeiss.com/microscopy/int/ } \\
\text { products/microscope-software/zen.html }\end{array}$ \\
\hline
\end{tabular}

\section{RESOURCE AVAILABILITY}

\section{Lead Contact}

Further information and requests for resources and reagents should be directed to and will be fulfilled by the Lead Contact, Nahum Sonenberg (nahum.sonenberg@mcgill.ca). 
Materials Availability

All reagents generated in this study are available from the Lead Contact with a completed Material Transfer Agreement.

Data and Code Availability

Original data have been deposited to Mendeley Data: https://doi.org/10.17632/rr89y6c68m.1

\section{METHOD DETAILS}

Cell culture, amino acid starvation, stimulation, and transient transfection HEK293T cells were maintained in Dulbecco's Modified Eagle Medium (DMEM) (Wisent) supplemented with $10 \%$ fetal bovine serum (FBS) (Wisent) and 1\% streptomycin-penicillin (Wisent) in a humidified atmosphere containing $5 \% \mathrm{CO}_{2}$ at $37^{\circ} \mathrm{C}$. For amino acid starvation, cells seeded $24 \mathrm{hr}$ before starvation were washed by pre-warmed PBS and incubated with amino acid-, leucine-, and threonine-free DMEM (manufactured by Wisent) supplemented with 10\% dialyzed FBS (GIBCO). For amino acid stimulation, a concentrated solution, which is prepared from individual amino acid powder, was added to the culture medium at the same concentration as in the complete DMEM (\#319-005-CL) (Wisent). Transient transfection of plasmids was performed using Lipofectamine 2000 (Invitrogen) according to the manufacturer's instructions.

\section{Lentivirus production}

HEK293T cells were transfected with lentiviral packaging plasmids (pLP-1, pLP-2, and pLP/VSVG) (Invitrogen) as well as a lentiviral expressing plasmid using Lipofectamine 2000 . After $48 \mathrm{hr}$, the medium containing lentiviral particles was collected, filtered using a $0.45 \mu \mathrm{m}$ syringe filter (Fisher Scientific), and immediately added to the HEK293T cells with $5 \mu \mathrm{g} / \mathrm{ml}$ of polybrene. The rest of the supernatant was stored at $-80^{\circ} \mathrm{C}$. After $24 \mathrm{hr}$, cells were selected using appropriate antibiotics.

\section{Immunoprecipitation}

Immunoprecipitation was performed as described previously (Kim et al., 2002) with minor modifications. Briefly, cells were washed by ice-cold PBS and collected by scraping in the lysis buffer (40 mM HEPES-KOH pH 7.5, $120 \mathrm{mM} \mathrm{NaCl,} 1 \mathrm{mM}$ EDTA, $50 \mathrm{mM} \mathrm{NaF}$, 0.3\% CHAPS, supplemented with a complete EDTA-free protease inhibitor tablet and phosphatase inhibitor cocktail). Pre-cleared lysates were incubated with primary antibody for $1 \mathrm{hr}$ at $4^{\circ} \mathrm{C}$, followed by incubated with Protein $\mathrm{G}$ or $\mathrm{A}$ agarose beads (Millipore) overnight at $4^{\circ} \mathrm{C}$. Beads were washed with wash buffer (lysis buffer supplemented with $50 \mathrm{mM} \mathrm{HEPES-KOH} \mathrm{pH} \mathrm{7.5,} 150 \mathrm{mM}$ $\mathrm{NaCl}$ ) for 10 min three times. Protein was eluted in 2x SDS sample buffer.

\section{Subcellular fractionation}

HEK293T cells were homogenized in lysis buffer (220 mM Mannitol, $68 \mathrm{mM}$ sucrose, $80 \mathrm{mM} \mathrm{KCl,} 0.5 \mathrm{mM} \mathrm{EGTA}, 2 \mathrm{mM} \mathrm{MgAc} 2,10 \mathrm{mM}$ HEPES-KOH pH 7.4, supplemented with a complete EDTA-free protease inhibitor tablet) using 26-gauge syringe needle for 10 strokes (total fraction). Homogenate was centrifuged at $600 \times \mathrm{g}$ for $10 \mathrm{~min}$ at $4^{\circ} \mathrm{C}$. Pellet was collected as a nuclear fraction and, the supernatant was centrifuged at $5,000 \times \mathrm{g}$ for $10 \mathrm{~min}$ at $4^{\circ} \mathrm{C}$. Pellet was collected as a mitochondrial (and small lysosomal) fraction, and the supernatant was centrifuged at $12,000 \times \mathrm{g}$ for $5 \mathrm{~min}$ at $4^{\circ} \mathrm{C}$. Pellet was collected as a pure lysosomal fraction, and the supernatant was collected as a crude cytoplasmic fraction. Pellets between each step were washed with lysis buffer, and the supernatant was centrifuged twice every step for reducing contamination.

\section{Immunofluorescence}

Cells seeded on the poly-L-lysine (Sigma) or fibronectin (Sigma)-coated coverslips were washed by pre-warmed PBS and fixed by pre-warmed 4\% paraformaldehyde (PFA) (FD Neurotechnologies, Inc.) for 15 min at RT. After washing 3 times with PBS, cells were permeabilized with PBS containing $0.05 \%$ Triton X-100 for 5 min at RT and blocked with blocking solution (PBS containing $10 \%$ FBS). Cells were incubated with primary antibodies (from 0.5 to $2: 100$ in blocking solution) overnight at $4^{\circ} \mathrm{C}$ and incubated with Alexa fluor (Thermo Fisher Scientific) secondary antibodies (1:1000 in blocking solution) for $1 \mathrm{hr}$ at RT in the darkness. Nuclei were stained with $2 \mu \mathrm{g} / \mathrm{ml}$ of Hoechst (Life Technologies) for $10 \mathrm{~min}$ at RT in the darkness. Cells were mounted onto the slide glass by using the Fluorescence Mounting Medium (Dako). Images were acquired using the 63X oil immersion lens of LSM 800 confocal microscope (Zeiss).

\section{Proximity ligation assay (PLA)}

PLA was performed using Duolink PLA (Sigma) according to the manufacturer's instructions. Briefly, cells seeded on the fibronectincoated coverslip were fixed and permeabilized as described above. Cells were blocked in Duolink blocking solution at $37^{\circ} \mathrm{C}$ for $1 \mathrm{hr}$ and incubated with primary antibodies for overnight at $4^{\circ} \mathrm{C}$. After washing with Wash Buffer A, cells were incubated with Duolink PLA probes at $37^{\circ} \mathrm{C}$ for $1 \mathrm{hr}$, followed by ligation at $37^{\circ} \mathrm{C}$ for $30 \mathrm{~min}$. The PLA signals were amplified using Amplification Buffer at $37^{\circ} \mathrm{C}$ for 100 min. After washing with Wash Buffer B, cells were mounted onto the slide glass. 


\section{$\infty$ CellPress}

\section{Molecular Cell

GTP-agarose pulldown assay

GTP-agarose pulldown assay was performed as described previously (Stafa et al., 2014) with minor modifications. Briefly, cells were lysed in lysis buffer (1\% Triton X-100 in PBS, supplemented with EDTA-free protease inhibitor tablet and phosphatase inhibitor cocktail). Cell lysates were incubated with GTP-agarose beads (Sigma) for $2 \mathrm{hr}$ at $4^{\circ} \mathrm{C}$. Beads were washed 3 times with lysis buffer and once with PBS. Protein was eluted in 2x SDS sample buffer.

\section{Polysome profiling}

Polysome profiling was performed as described (Gandin et al., 2014) with minor modifications. Briefly, HEK293T cells at 80\%-90\% confluency were treated with $100 \mu \mathrm{g} / \mathrm{ml}$ of cycloheximide $(\mathrm{CHX})$ for $5 \mathrm{~min}$ at $37^{\circ} \mathrm{C}$. Cells were placed on ice, washed twice with icecold PBS containing $100 \mu \mathrm{g} / \mathrm{ml}$ of $\mathrm{CHX}$, and suspended in hypotonic buffer $(5 \mathrm{mM} \mathrm{Tris-HCl} \mathrm{pH} \mathrm{7.4,} 1.5 \mathrm{mM} \mathrm{KCl}, 2.5 \mathrm{mM} \mathrm{MgCl}$, $100 \mu \mathrm{g} / \mathrm{ml}$ of $\mathrm{CHX}, 200 \mathrm{u} / \mathrm{ml}$ of RNase inhibitor, $2 \mathrm{mM} \mathrm{DTT}$, supplemented with EDTA-free protease inhibitor tablet). Cells were lysed by adding detergent mixture ( $0.5 \%$ of Triton X-100 and $0.5 \%$ of sodium deoxycholate) and vortexed for $5 \mathrm{~s}$. The amount of RNA in the supernatant was measured using NanoDrop 2000 (Thermo Fisher Scientific), and the equal amount of each sample was loaded onto $10 \%-50 \%$ sucrose gradient containing $20 \mathrm{mM} \mathrm{HEPES}-\mathrm{KOH} \mathrm{pH} \mathrm{7.6,100} \mathrm{mM} \mathrm{KCl,} 5 \mathrm{mM} \mathrm{MgCl}, 10 \mu \mathrm{g} / \mathrm{ml} \mathrm{of} \mathrm{CHX,10} \mathrm{u/ml} \mathrm{RNase}$ inhibitor, supplemented with EDTA-free protease inhibitor tablet. Gradients were balanced and centrifuged at 36,000 rpm for $2 \mathrm{hr}$ at $4^{\circ} \mathrm{C}$ using SW $40 \mathrm{Ti}$ rotor in Optima L-80 XP ultracentrifuge (Beckman Coulter). Absorbance at $254 \mathrm{~nm}$ was measured using TracerDAQ (MicroDAQ) from lower to higher sedimentation of sucrose gradients. 
Molecular Cell, Volume 81

Supplemental Information

Mitochondrial Threonyl-tRNA Synthetase TARS2 Is

Required for Threonine-Sensitive mTORC1 Activation

Sung-Hoon Kim, Jung-Hyun Choi, Peng Wang, Christopher D. Go, Geoffrey G. Hesketh, Anne-Claude Gingras, Seyed Mehdi Jafarnejad, and Nahum Sonenberg 
Figure S1.

A

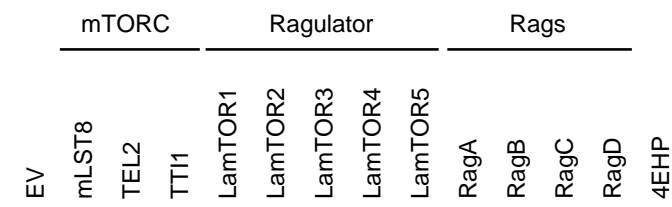

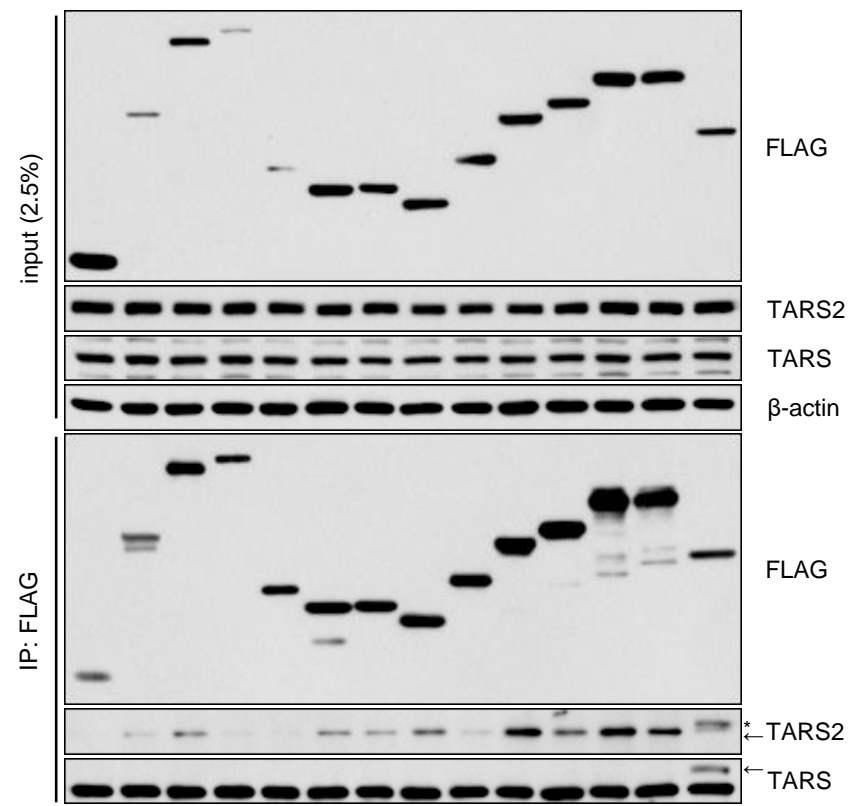

C
TOM20

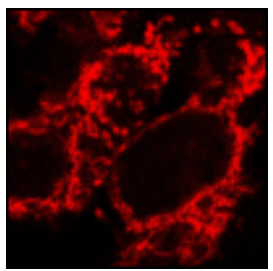

TOM20

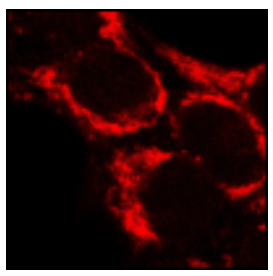

D
B

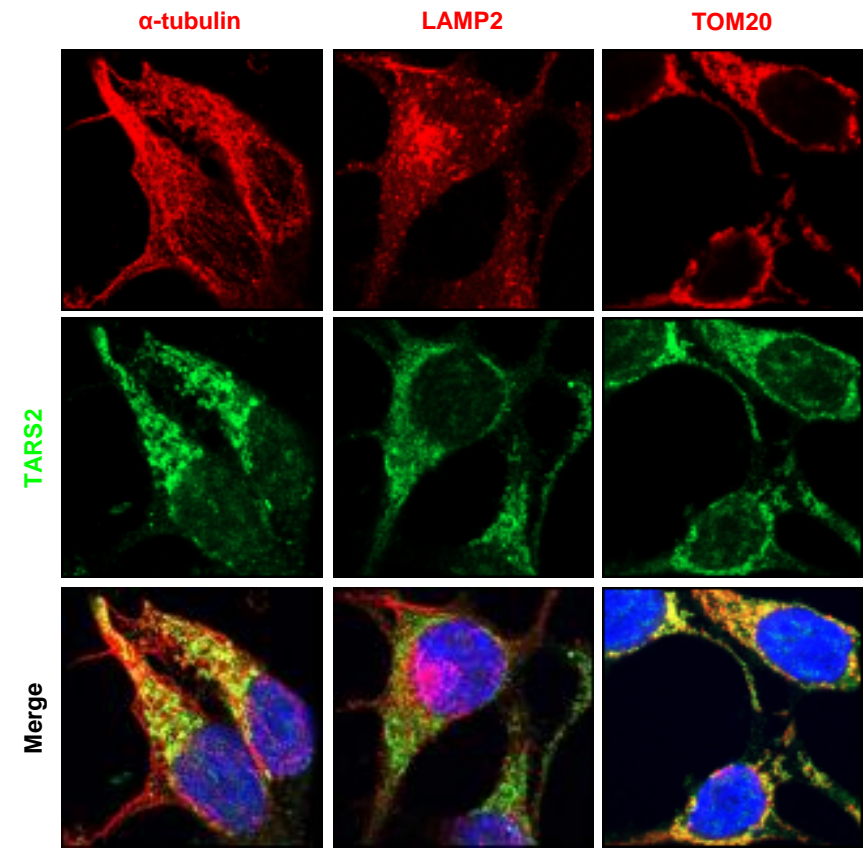

a-tubulin

LAMP2

TOM20
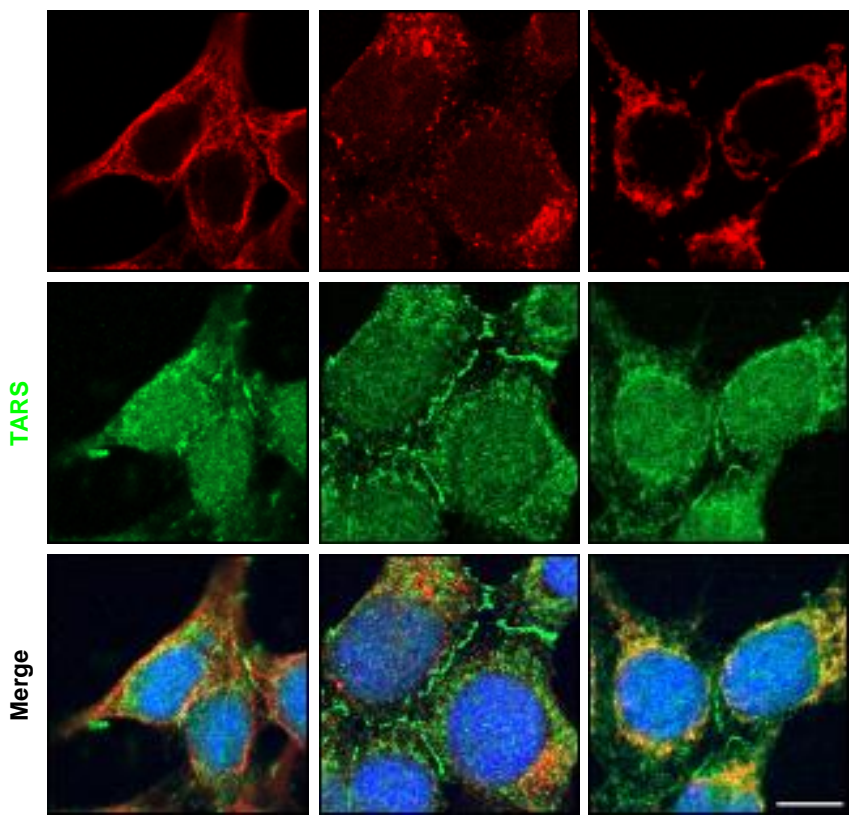

Hoechst

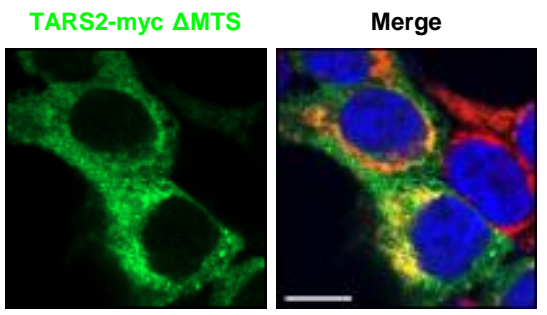

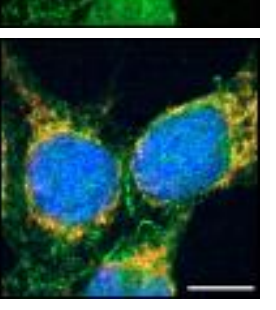

TARS2-myc FL

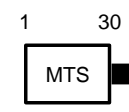


Figure S1. Related to Figure 1

(A) Interaction of TARS2 and TARS with AA-sensing machinery of mTORC1. HeLa cells were transfected with the indicated plasmids and used for IP with an anti-FLAG antibody. Arrows indicate TARS2 or TARS-specific band and an asterisk indicates TARS pulled down by cross-reactivity. EV (empty vector).

(B) Subcellular localization of TARS2 and TARS. a-tubulin (cytoskeleton), LAMP2 (lysosome), TOM20 (mitochondrion). Scale bar $=10 \mu \mathrm{m}$.

(C) Localization of full-length (TARS2-myc FL) or non-mitochondrial (TARS2-myc $\Delta M T S$ ) TARS2. Scale bar $=10 \mu m$.

(D) Schematic representation of TARS2 mutants used in panel $(C)$ with AA number is shown. 


\section{Figure S2.}

A

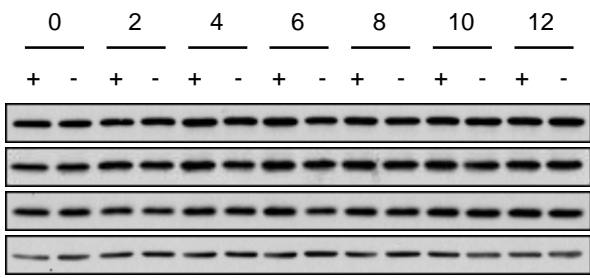

C

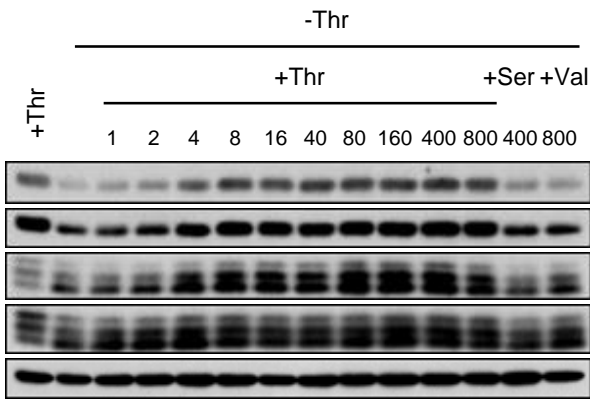

D

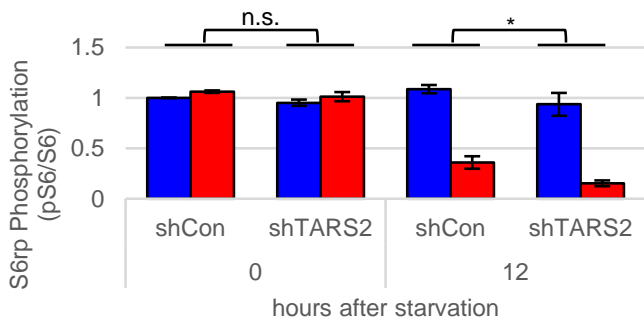

F

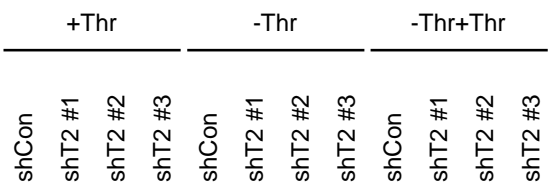

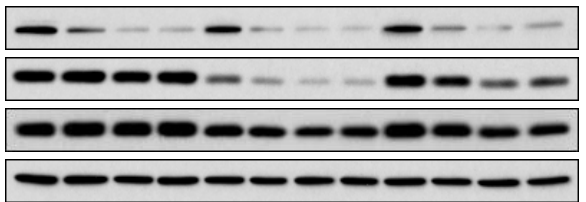

TARS2

pS6

(S240/244)

S6

$\beta$-actin

(S240/244)

p4E-BP1

(T37/46)

$\beta$-actin

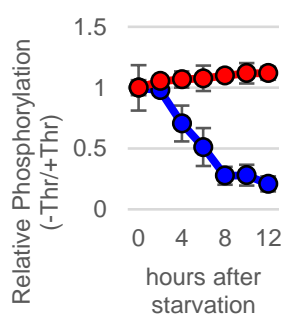

B

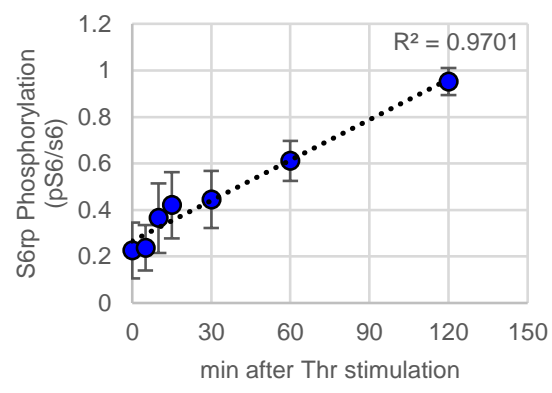

$\longrightarrow$ S6 $\longrightarrow$ AKT

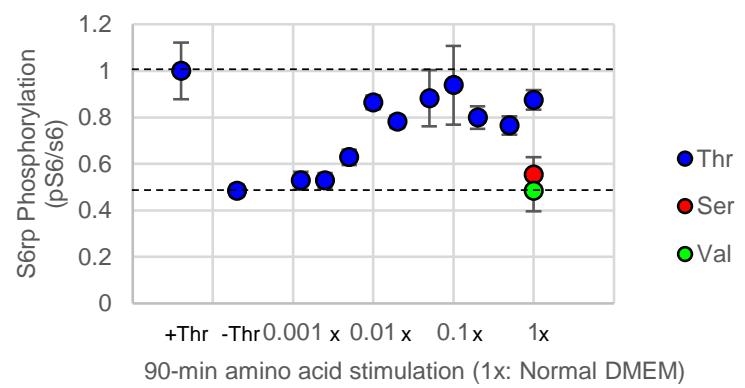

E

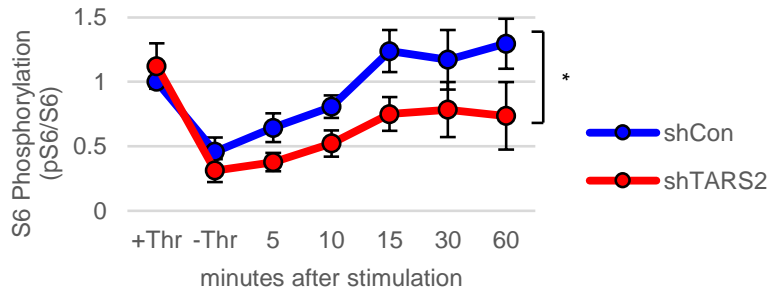

G

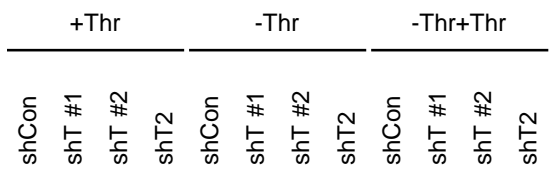

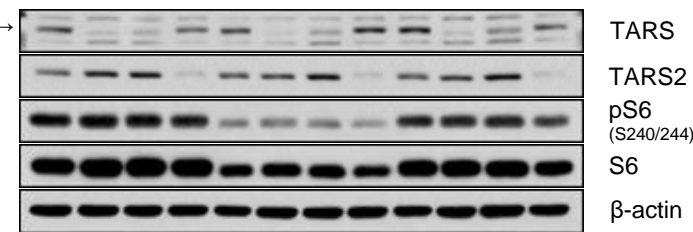

minutes after stimulation

H

\begin{tabular}{|c|c|c|}
\hline+ Thr & -Thr & $-\mathrm{Thr}+\mathrm{Th}$ \\
\hline+ & + & + \\
\hline+ & + & + \\
\hline - - & $0-$ & $-6-$ \\
\hline-- & 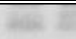 & -1 \\
\hline 5 & $\rightarrow$ & $-\infty=$ \\
\hline-1 & $-=$ & $=-$ \\
\hline-- & $-a$ & -1 \\
\hline & -7 & $-1-$ \\
\hline
\end{tabular}

shCon

shTARS2

TARS2

pS6

(S240/244)

S6

pelF $2 \alpha$

(S51)

elF2a

ATF4

Sestrin2

$\beta$-actin

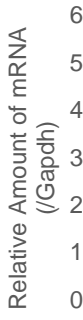

$\mu \mathrm{M}, 90 \mathrm{~min}$

+Thr

-Thr

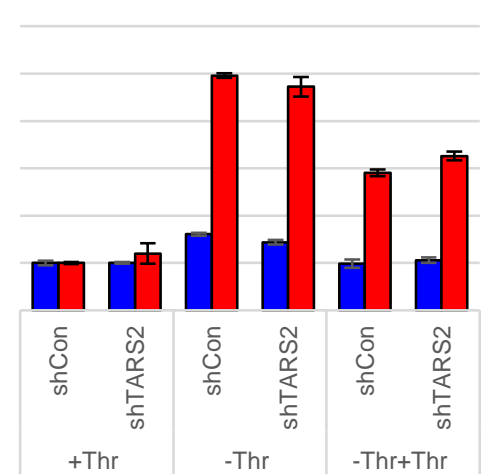

口Atf4 口Sestrin2 
Figure S2. Related to Figure 2

(A) mTORC2 activity under starved (-) versus normal Thr levels (+). (Left) PAKT S473 was used as an mTORC2 activity marker. (Right) Mean \pm SEM of pS6 or pAKT from 3 independent experiments is shown.

(B) Recovery of mTORC1 activity by Thr stimulation. Mean \pm SEM of pS6 from 3 independent experiments and the $\mathrm{R}^{2}$ value, a determinant coefficient of linear regression, are shown.

(C) Recovery of mTORC1 activity by stimulation of threonine (Thr), serine (Ser), or valine (Val). HEK293T cells starved of Thr for $15 \mathrm{hr}$ (-Thr) and then supplemented with a range of concentrations from 1 to $800 \mu \mathrm{M}$ Thr $(-\mathrm{Thr}+\mathrm{Thr}), 400 \mu \mathrm{M}$ Ser (+Ser), or $800 \mu \mathrm{M} \mathrm{Val} \mathrm{(+Val)} \mathrm{for} 90$ min. (Right) Mean \pm SEM of pS6 from 3 independent experiments is shown. 1x indicates $800 \mu \mathrm{M}$ of Thr, $400 \mu \mathrm{M}$ of Ser, and $800 \mu \mathrm{M}$ of Val.

(D) mTORC1 activity in TARS2 knockdown cells (shTARS2) under Thr starvation. Mean \pm SEM of pS6 from 3 independent experiments is shown. n.s., not significant, * $p<0.05$ (one-way ANOVA with Dunnett's posthoc test).

(E) Recovery of mTORC1 activity in shTARS2 by Thr stimulation. Mean \pm SEM of pS6 from 3 independent experiments is shown. * $p<0.05$ (one-way ANOVA with Dunnett's posthoc test).

(F) mTORC1 activity in TARS2 knockdown cells under starvation and stimulation of Thr. HEK293T cells stably expressing control shRNA (shCon) and shTARS2 (shT2) were starved of Thr for $15 \mathrm{hr}$ (-Thr) and then stimulated by $800 \mu \mathrm{M}$ Thr for $1 \mathrm{hr}(-\mathrm{Thr}+\mathrm{Thr})$.

(G) mTORC1 activity in TARS knockdown cells (shT) under starvation and stimulation of Thr. Cells were subjected to the same treatment as described in panel $(F)$. An arrow indicates TARS.

(H) elF2a-ATF4-Sestrin2 pathway in shTARS2 under starvation and stimulation of Thr. (Left) Cells were subjected to the same treatment as described in panel (F). (Right) Mean \pm SEM of mRNA levels from 3 technical replicates. 


\section{Figure S3.}

A

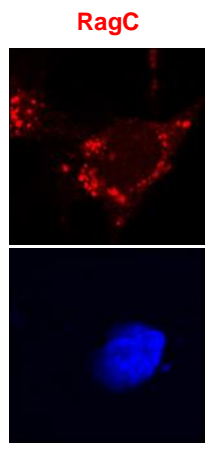

Hoechst
TARS2-myc

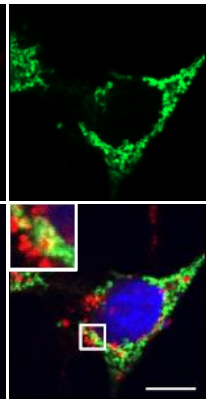

Merge
B

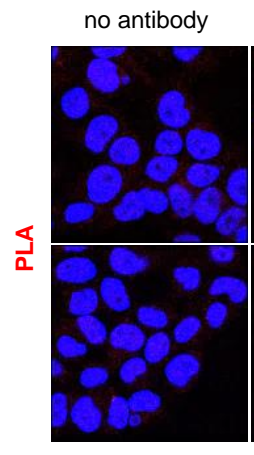

RagC only

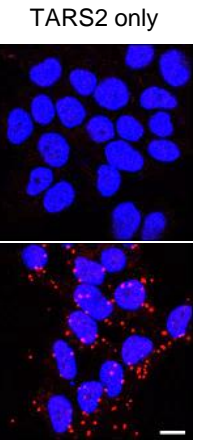

TARS2 + RagC
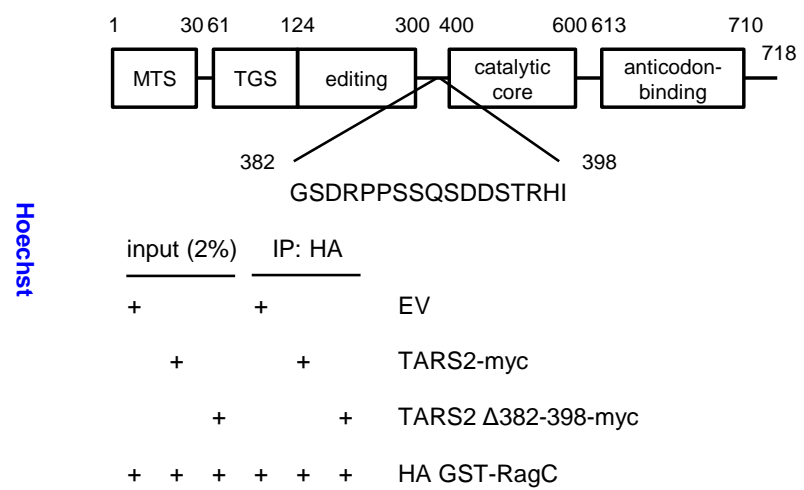

EV

TARS2-myc

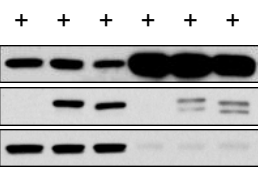

TARS2 $\Delta 382-398-m y c$

HA GST-RagC

$\mathrm{HA}$

myc

$\beta$-actin

F

\begin{tabular}{|c|c|c|c|c|c|c|c|c|c|}
\hline & 275 & 281 & 306 & 308 & 309 & 315 & 340 & 341 & 370 \\
\hline RagC & $\mathrm{S}$ & $\mathrm{S}$ & $\mathrm{S}$ & $\mathrm{S}$ & $\mathrm{A}$ & $\mathrm{M}$ & $\mathrm{I}$ & $\mathrm{L}$ & $\mathrm{G}$ \\
\hline RagD & $\mathrm{T}$ & $\mathrm{T}$ & $\mathrm{A}$ & $\mathrm{T}$ & $\mathrm{P}$ & $\mathrm{T}$ & $\mathrm{F}$ & $\mathrm{V}$ & $\mathrm{R}$ \\
\hline
\end{tabular}

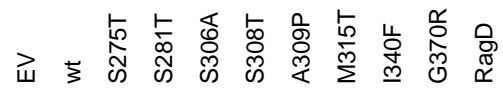

HA GST-RagC/RagD

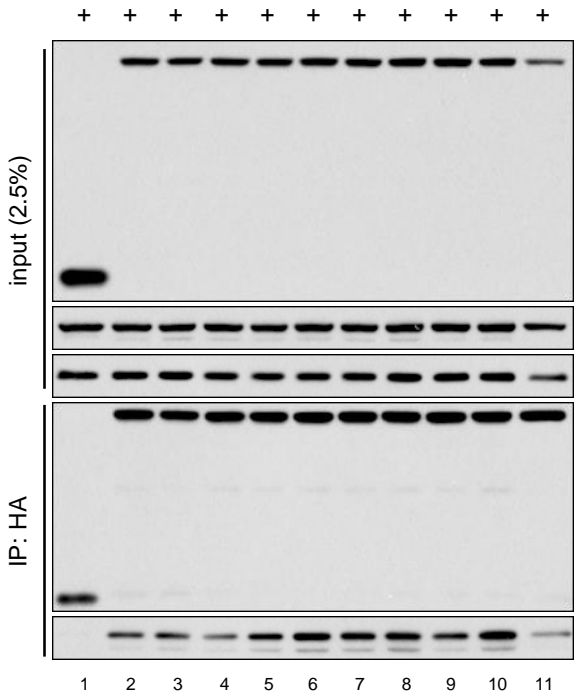

TARS2-myc

$\beta$-actin

HA

myc

-

$\begin{array}{llllllllllll}1 & 2 & 3 & 4 & 5 & 6 & 7 & 8 & 9 & 10 & 11 & 12\end{array}$ lane

E

61

$370 \quad 399$

G domain

243

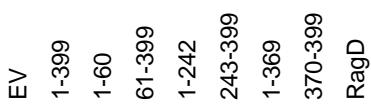

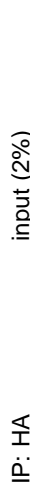

HA GST-RagC/RagD

TARS2-myc

$+++++++$

จे
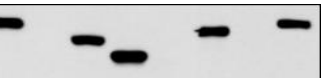

HA

0

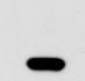

$-\infty-\infty-\infty-$

20

$-\infty-\infty-\infty-\infty$

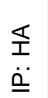

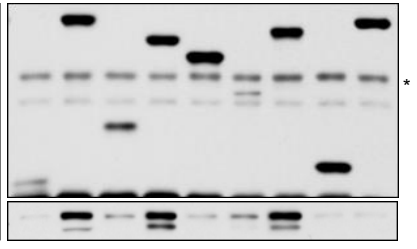

myc

$\beta$-actin

HA
G

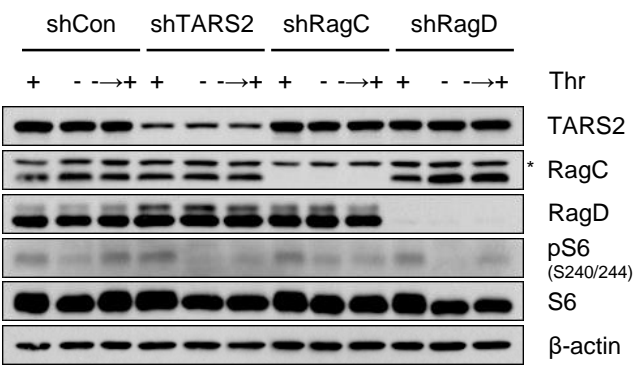


Figure S3. Related to Figure 3

(A) Co-localization of TARS2 with RagC. Nuclei were stained by Hoechst (blue). White squares in the merged images are enlarged in the upper left. Scale bar $=10 \mu \mathrm{m}$.

(B) Proximal interaction of TARS2 with RagC. Nuclei were stained by Hoechst (blue). Scale bar $=10 \mu \mathrm{m}$.

(C) RagC-interacting region of TARS2. (Upper) Schematic representation of functional domains of TARS2 is shown with AA number. MTS, ThrRS-GTPase-SpoT domain (TGS), editing domain, catalytic core domain, and the anticodonbinding domain are indicated. (Lower) HEK293T cells were transfected with truncation mutants of TARS2 or full-length TARS and used for IP with an anti-HA antibody. An asterisk indicates the light IgG chain. EV (empty vector).

(D) TARS2301-381 is sufficient for the interaction with RagC. (Upper) Schematic representation of a mitochondrial TARS2-exclusive segment is shown. (Lower) HEK293T cells were transfected with plasmids expressing full-length TARS2 (TARS2-myc) or TARS2 lacking 382-398 (TARS2 $\Delta 382-398-m y c)$ and used for IP with an anti-HA antibody. EV (empty vector).

(E) TARS2-interacting region of RagC. (Upper) Schematic representation of functional domains of RagC is shown with AA number. The $\mathrm{G}$ domain responsible for guanine nucleotide-binding and the C-terminal region, which is highly conserved in RagD, are indicated by a white box and a thick line, respectively. (Lower) HEK293T cells were transfected with truncation mutants of RagC or full-length RagD and used for IP with an anti-HA antibody. An asterisk indicates the heavy IgG chain. EV (empty vector).

(F) Preferential interaction of TARS2 with RagC. (Upper) The difference in $\mathrm{AA}_{1}$ sequence between RagC $243-369$ and the corresponding region in RagD. (Lower) HEK293T cells were transfected with wild-type (wt), mutant RagC, or full-length RagD and used for IP with an anti-HA antibody.

(G) Recovery of mTORC1 activity in RagC knockdown cells. Cells stably expressing the indicated shRNA were starved of Thr for $15 \mathrm{hr}(-)$ and then supplemented with $800 \mu \mathrm{M}$ Thr for $1 \mathrm{hr}(-\rightarrow+)$. An asterisk indicates a non-specific band. 
Figure S4.

A

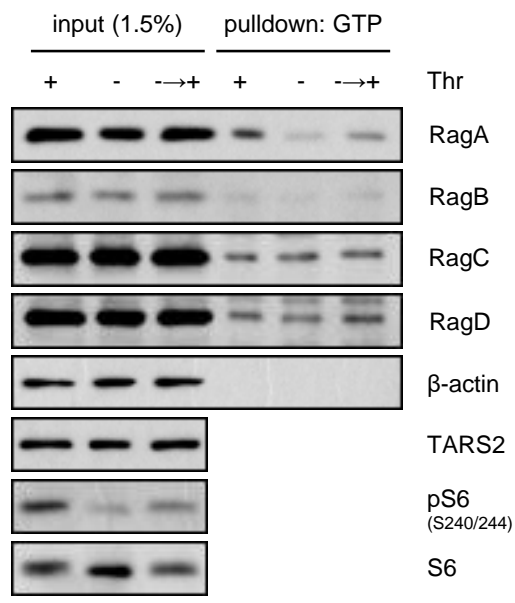

C

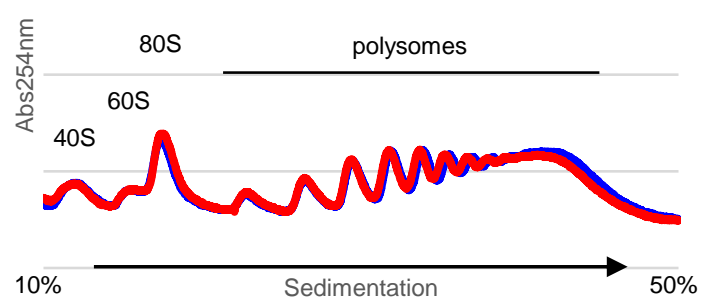

$\longrightarrow$ shCon +Thr $=$ shTARS2 + Thr

\section{B}
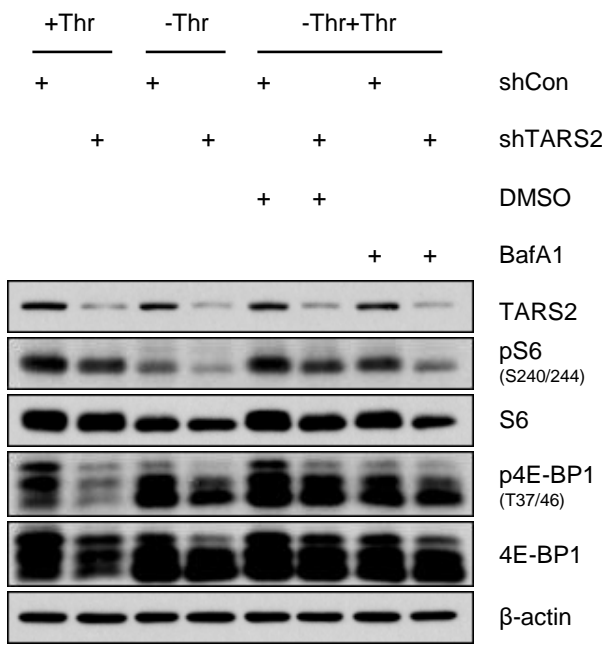

TARS2

pS6

(S240/244)

56

p4E-BP1

(T37/46)

4E-BP1

$\beta$-actin

D

$80 \mathrm{~S}$

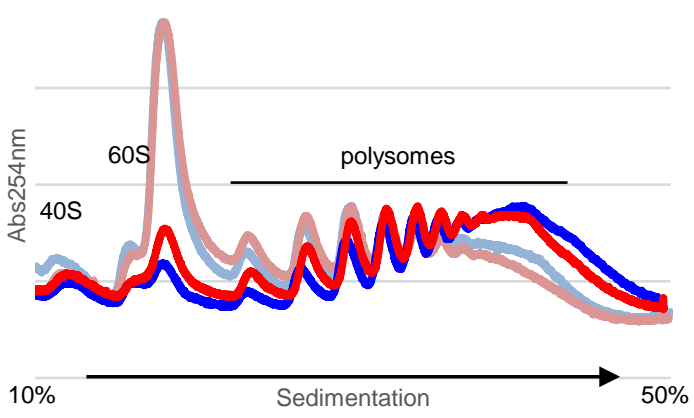

shCon -Thr shTARS2 -Thr

shCon -Thr+Thr $\longrightarrow$ shTARS2 -Thr+Thr 
Figure S4. Related to Figure 4

(A) GTP-loading of Rags under starvation and stimulation of Thr. Cells were starved of Thr for $15 \mathrm{hr}(-)$ and then stimulated by $800 \mu \mathrm{M}$ Thr for $1 \mathrm{hr}(-\rightarrow+)$. Cells were subjected to GTP-agarose pulldown assay.

(B) GTP-loading of Rags with or without Bafilomycin A1 (BafA1) in TARS2 knockdown cells. Cells were subjected to the same treatment as described in panel (A) but treated with vehicle (DMSO) or BafA1 $1 \mathrm{hr}$ before the addition of Thr. (C) Representative polysome profile under normal Thr condition. Small ribosome subunit (40S), large ribosome subunit (60S), monosome (80S), and polyribosome (polysomes) fractions are shown.

(D) Representative polysome profile under Thr-starved (-Thr, light color) or Thr-stimulated (-Thr+Thr, dark color) condition. 